\title{
Lysine acetyltransferase Tip60 acetylates the APP adaptor Fe65 to increase its transcriptional activity
}

Probst, Sabine ; Riese, Florian ; Kägi, Larissa ; Krüger, Maik ; Russi, Natalie ; Nitsch, Roger M ; Konietzko, Uwe

\begin{abstract}
Proteolytic processing of the amyloid precursor protein (APP) releases the APP intracellular domain (AICD) from the membrane. Bound to the APP adaptor protein Fe65 and the lysine acetyltransferase (KAT) Tip60, AICD translocates to the nucleus. Here, the complex forms spherical condensates at sites of endogenous target genes, termed AFT spots (AICD-Fe65-Tip60). We show that loss of Tip60 KAT activity prevents autoacetylation, reduces binding of Fe65 and abolishes Fe65-mediated stabilization of Tip60. Autoacetylation is a prerequisite for AFT spot formation, with KAT-deficient Tip60 retained together with Fe65 in speckles. We identify lysine residues 204 and 701 of Fe65 as acetylation targets of Tip60. We do not detect acetylation of AICD. Mutation of Fe65 K204 and K701 to glutamine, mimicking acetylation-induced charge neutralization, increases the transcriptional activity of Fe65 whereas Tip60 inhibition reduces it. The lysine deacetylase (KDAC) class III Sirt1 deacetylates Fe65 and pharmacological modulation of Sirt1 activity regulates Fe65 transcriptional activity. A second acetylation/deacetylation cycle, conducted by CBP and class I/II KDACs at different lysine residues, regulates stability of Fe65. This is the first report describing a role for acetylation in the regulation of Fe65 transcriptional activity, with Tip60 being the only KAT tested that supports AFT spot formation.
\end{abstract}

DOI: https://doi.org/10.1515/hsz-2020-0279

Posted at the Zurich Open Repository and Archive, University of Zurich

ZORA URL: https://doi.org/10.5167/uzh-205365

Journal Article

Published Version

Originally published at:

Probst, Sabine; Riese, Florian; Kägi, Larissa; Krüger, Maik; Russi, Natalie; Nitsch, Roger M; Konietzko, Uwe (2021). Lysine acetyltransferase Tip60 acetylates the APP adaptor Fe65 to increase its transcriptional activity. Biological Chemistry, 402(4):481-499.

DOI: https://doi.org/10.1515/hsz-2020-0279 


\section{Sabine Probst, Florian Riese, Larissa Kägi, Maik Krüger, Natalie Russi, Roger M. Nitsch} and Uwe Konietzko*

\section{Lysine acetyltransferase Tip60 acetylates the APP adaptor Fe65 to increase its transcriptional activity}

https://doi.org/10.1515/hsz-2020-0279

Received August 10, 2020; accepted November 18, 2020; published online December 2, 2020

\begin{abstract}
Proteolytic processing of the amyloid precursor protein (APP) releases the APP intracellular domain (AICD) from the membrane. Bound to the APP adaptor protein Fe65 and the lysine acetyltransferase (KAT) Tip60, AICD translocates to the nucleus. Here, the complex forms spherical condensates at sites of endogenous target genes, termed AFT spots (AICD-Fe65-Tip60). We show that loss of Tip60 KAT activity prevents autoacetylation, reduces binding of Fe65 and abolishes Fe65-mediated stabilization of Tip60. Autoacetylation is a prerequisite for AFT spot formation, with KAT-deficient Tip60 retained together with Fe65 in speckles. We identify lysine residues 204 and 701 of Fe65 as acetylation targets of Tip60. We do not detect acetylation of AICD. Mutation of Fe65 K204 and K701 to glutamine, mimicking acetylation-induced charge neutralization, increases the transcriptional activity of Fe65 whereas Tip60 inhibition reduces it. The lysine deacetylase (KDAC) class III Sirt1 deacetylates Fe65 and pharmacological modulation of Sirt1 activity regulates Fe65 transcriptional activity. A second acetylation/deacetylation cycle, conducted by CBP and class I/II KDACs at different lysine residues, regulates stability of Fe65. This is the first report describing a role for acetylation in the regulation of Fe65 transcriptional activity, with Tip60 being the only KAT tested that supports AFT spot formation.
\end{abstract}

Keywords: acetylation; AFT spots; AICD; CBP; Sirt1; transcription assay.

*Corresponding author: Uwe Konietzko, Institute for Regenerative Medicine (IREM), University of Zurich Campus Schlieren, Wagistrasse 12, $\mathrm{CH}-8952$ Schlieren, Switzerland,

E-mail: uwe.konietzko@irem.uzh.ch. https://orcid.org/0000-00026897-232X

Sabine Probst, Florian Riese, Larissa Kägi, Maik Krüger, Natalie Russ and Roger M. Nitsch, Institute for Regenerative Medicine (IREM), University of Zurich Campus Schlieren, Wagistrasse 12, CH-8952 Schlieren, Switzerland

\section{Introduction}

The amyloid precursor protein (APP) is a type I transmembrane glycoprotein, causally involved in Alzheimer's disease (AD). Sequential proteolytic cleavage of APP via the amyloidogenic pathway releases the beta amyloid peptide $(A \beta)$, which aggregates into oligomeric structures that disrupt synaptic function in AD (Kang et al. 1987; Selkoe and Hardy 2016). Intramembraneous cleavage by the $y$-secretase complex also releases the APP intracellular domain (AICD) from the membrane. Together with bound cytosolic proteins, AICD can signal to the nucleus to regulate transcription (Konietzko 2012). First evidence for such a function originated from experiments using Gal4based luciferase assays with the Gal4-DNA binding domain (DBD) inserted in front of the AICD sequence in APP (Cao and Sudhof 2001). These experiments also revealed a prominent role for the AICD-binding protein Fe65 (Duilio et al. 1991) and further discovered the involvement of the KAT Tip60.

Fe65 is a multi-domain protein, containing a polyprolinebinding WW domain and two phosphotyrosine-binding (PTB) domains. These are bracketed and connected by sequences of unknown structure. Fe65 sequence analysis on the Phyre2 server (Kelley et al. 2015) predicts that $64 \%$ of the sequence is disordered. With its different domains, Fe65 can scaffold protein complexes. It connects transmembrane proteins such as the APP family or the lipoprotein receptor family to intracellular proteins, including mammalian enabled (Mena), Abelson non-receptor tyrosine kinase (c-Abl), Tip60, nucleosome assembly factor SET, ras protein Dexras 1 and 14-3-3y (reviewed in (Konietzko 2012)). We have recently shown that Fe65 and Tip60 associate in the cytoplasm and promote each other's nuclear translocation (Probst et al. 2020). Co-expression of APP or AICD with Fe65 and Tip60 in various cell lines and primary cells leads to the formation of spherical spots in the nucleus designated AFT spots according to the involved proteins (Goodger et al. 2009; von Rotz et al. 2004). Nuclear AFT spots co-localize with the Notch intracellular domain (NICD) that has transcriptional 
activity and also localize to the gene loci of APP and KAI1, two reported target genes of AICD (Konietzko et al. 2010; Pardossi-Piquard and Checler 2012). The liquid-like properties of AFT spots, such as their spherical shape and the ability to flow and fuse, are highly reminiscent other nuclear phase-separated condensates that contain components of the transcriptional machinery (Cho et al. 2018). Together with further evidence, we postulated that AFT spots correspond to nuclear transcription factories (Konietzko et al. 2010). Several studies using APP-Gal4-based assays reported a dramatic enhancement of transcriptional activity when Fe65 was co-expressed (Cao and Sudhof 2001; Konietzko et al. 2019; Wiley et al. 2007; Zambrano et al. 1997). We have recently acquired data providing strong evidence that Fe65 per se is a transcription factor, whose nuclear signaling activity is regulated by the rate of APP cleavage (Probst et al. 2020). The central parts of Fe65, including the WW and PTB1 domains are transcriptionally active, whereas the $\mathrm{N}$ - and $\mathrm{C}$-terminal parts, including the PTB2 domain have a repressive function. Less clear is the function of the Tip60 KAT in APP-mediated nuclear signaling.

Acetylation is a reversible posttranslational modification of lysine residues at their $\varepsilon$-amino group. Acetylation by KATs removes the positive charge of the lysine residue leading to structural changes in proteins. Lysine deacetylases (KDACs) can reverse this reaction. Acetylation was first discovered as a posttranslational modification of histones with an activating effect on gene transcription (Grunstein 1997). Acetylation decreases the affinity of histones to DNA, providing access for transcription factors (Lee et al. 1993). Besides histones, an increasing number of non-histone proteins have been found to be acetylated, with proteomic screens identifying thousands of lysine acetylation sites in the proteome (Choudhary et al. 2009; Zhao et al. 2010).

KATs are grouped into different families based on their catalytic domains. The major families are GNATs (Gcn5 N-acetyltransferases), CBP/p300 and MYST (Lee and Workman 2007). The MYST family is conserved from yeast to humans and includes Tip60, MOZ (monocytic leukaemia zinc finger protein), $\mathrm{hMOF}$ (human orthologue of Drosophila Mof, males-absent-on-the-first), HBO1 (HAT bound to ORC1, replication origin recognition complex 1) and MORF (MOZ-related factor). Tip60 (the 60-kDa HIV-Tat interactive protein; KAT5) was identified in a screen for HIV Tatinteracting proteins (Kamine et al. 1996). MYST acetyltransferases contain a MYST domain consisting of a zinc finger and an acetyl-CoA binding site (Roth et al. 2001), which are present in all three Tip60 splice variants (Sapountzi et al. 2006). Tip60 has been shown to acetylate core histones H2A, H3 and H4 (Kimura and Horikoshi 1998), as well as a number of transcription factors, such as the androgen receptor (Brady et al. 1999), the kinase ATM (ataxia telangiectasia mutated) (Sun et al. 2005), p53 (Sykes et al. 2006; Tang et al. 2006) and HIF1A (Perez-Perri et al. 2016). Tip60 can undergo autoacetylation and several autoacetylated lysine residues have been suggested, with K327 most consistently reported. Moreover, K327 is conserved between all members of the MYST family (Cui et al. 2015; Donmez et al. 2010; Peng et al. 2012; Tang et al. 2006; Xiao et al. 2014; Yang et al. 2012; Yi et al. 2014). Mutagenesis of lysine 327 to arginine, a mimetic substitute of a non-acetylatable lysine, causes loss of autoacetylation and a decrease in KAT activity, indicating that Tip60 autoacetylation at K327 is important for catalytic activity (Peng et al. 2012; Xiao et al. 2014; Yang et al. 2012). Tip60 is located to nuclear speckles and undergoes nucleocytoplasmic cycling (Konietzko et al. 2010). Some APP-interacting proteins, such as Jip1b and Fe65L2, colocalize together with Tip60 in speckles, both showing no transcriptional activity (Probst et al. 2020; von Rotz et al. 2004).

As described above, Fe65 supports Tip60 nuclear translocation and induces re-location to AFT spots, as well as possesses transcriptional activity. We studied how Tip60 KAT activity influences the formation of nuclear AFT spots and how it affects transcriptional activity. Our data suggest that the KAT activity is required for Tip60 autoacetylation, which is essential for the formation of AFT spots. Additionally, we found that Fe65 is acetylated by Tip60 at lysine residues 204 and 701 and that this acetylation increases the transcriptional activity of Fe65. We also identified a separate acetylation/deacetylation cycle, involving CBP and class I/II KDACs acting at different lysine residues that regulates the stability of Fe65.

\section{Results}

\section{AFT spot formation requires Tip60 KAT activity}

Tip60 is a lysine acetyl transferase of the MYST family. Together with APP and Fe65 it forms spherical nuclear complexes, designated AFT spots (von Rotz et al. 2004). To analyze whether the Tip60 KAT activity is required for AFT spot formation, we introduced two mutations (Q377E and G380E), reported to disrupt KAT activity (Ikura et al. 2000). Wildtype (wt) and KAT-deficient ( $\triangle$ KAT) CFP-Tip60 were co-transfected with APP-Cit and HA-Fe65 into HEK293 cells 
and assessed by confocal microscopy (Figure 1A). While wt Tip60 forms AFT spots together with APP and Fe65, the mutant Tip60 is localized in nuclear speckles and Fe65 and APP co-localize in extranuclear sites. We replicated this experiment with myc-tagged Tip60 constructs, and confirmed that only wt but not mutant Tip60 enables AFT spot formation (Figure S1A). Nuclear spots are clearly visible by fused fluorescent proteins (CFP and Cit), but antibody-mediated staining is less clear because of restricted staining in the nuclear environment (Konietzko et al. 2010; von Rotz et al. 2004). A similar effect of wt and $\triangle$ KAT Tip60 is also observed in the mouse neuroblastoma cell line N2a (Figure 2E), demonstrating that the role of the Tip60 KAT activity on AFT spot formation is neither cell line nor species dependent. With co-expression of APP and $\triangle$ KAT Tip60, Fe65 is bound to APP and no Fe65 is detected in the nucleus. Confocal microscopy of HEK293 cells coexpressing mCherry-Fe65 and CFP-Tip60 without APP, revealed their co-localization in nuclear spots in the case of wt Tip60, whereas the $\triangle$ KAT Tip60 resides in nuclear speckles together with Fe65 (Figure S1B).

We further performed a Bimolecular Fluorescence Complementation (BiFC)-based assay (Riese et al. 2013), where the YFP halves YN155 and YC155 were fused to Tip60 and APP respectively. A BiFC signal is only obtained when APP and Tip60 are brought into proximity by binding to Fe65, as it occurs in AFT spots. We observed that only wt, but not $\triangle$ KAT Tip60, generated a BiFC signal that localized to nuclear spots (Figure 1B). Quantification of the BiFC signal by flow cytometry showed a significant reduction when $\triangle$ KAT Tip60 was co-transfected (Figure 1C). These experiments demonstrate that the KAT activity of Tip60 is required for the formation of AFT spots.

\section{Tip60 acetylates itself and Fe65 but not APP}

We analyzed whether the Tip60 KAT activity is required for the acetylation of proteins involved in the formation of AFT spots. We transfected streptavidin-binding peptide (SBP)-myc-Fe65, APP-Cit, and wt or $\triangle$ KAT myc-Tip60 into HEK293 cells. We performed Dynabeads-Streptavidinbased pulldown via the SBP tag (Feilen et al. 2017; Kohli et al. 2012), to isolate SBP-myc-Fe65 from the cell lysate, and could co-precipitate myc-Tip60, APP-Cit and its cleavage product AICD-Cit in the eluate (Figure 2A). Glyceraldehyde 3-phosphate dehydrogenase (GAPDH) on the contrary, was only detected in the lysate but not in the eluate. Transfected Tip60 and Fe65 are detected by the c-myc antibody, while APP-Cit and AICD-Cit are detected by the GFP antibody. An acetylated-lysine antibody

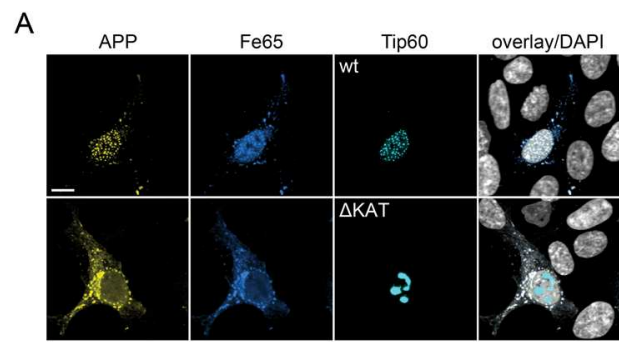

B

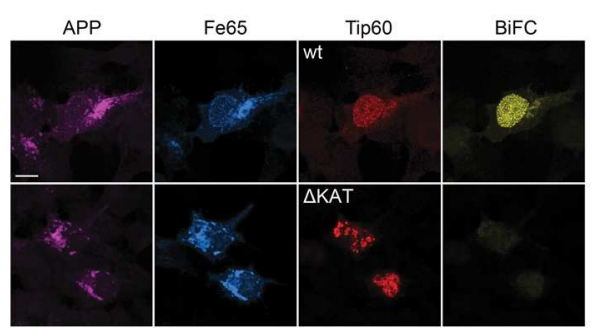

C

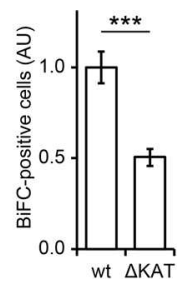

Figure 1: AFT spot formation requires Tip60 KAT activity.

(A) Confocal microscopy shows that co-expression of APP-Cit and HA-Fe65 with wt CFP-Tip60 (top row) leads to AFT spot formation, while the $\triangle$ KAT Tip60 (bottom row) stays in speckles in HEK293 cells. (B and C) Co-expression of APP-YC155, HA-Fe65, and wt or $\triangle$ KAT mycTip60-YN155. BiFC of the yellow fluorescent protein fusion fragments YC155 and YN155 only occurs upon Fe65-mediated nuclear translocation of AICD and interaction with wt Tip60, as detected by (B) confocal microscopy and (C) flow cytometry. Mean \pm SEM of $n=11-12$ are shown (two-tailed $t$-test). Scale bars: $10 \mu \mathrm{m}$.

detects multiple bands in cell lysates and enables analysis of acetylation levels in co-precipitated proteins in the eluate. Wt myc-Tip60 autoacetylates itself and additionally acetylates SBP-myc-Fe65. In contrast, even with longer exposure, neither an acetyl-lysine signal for APP-Cit nor for AICD-Cit was detectable. The $\triangle$ KAT Tip60 co-precipitates with Fe65 at lower levels and no acetyllysine signal can be detected for Tip60 or Fe65. We repeated the experiment with Cit-AICD where Cit is fused $\mathrm{N}$-terminally to the AICD peptide (Figure S2A). Fe65 coprecipitated Cit-AICD and myc-Tip60, but we could not detect acetylation of AICD, even with longer exposure. We next performed pulldown experiments with SBP-Fe65 and Tip60 without co-expression of APP. We confirmed that only wt, but not $\triangle$ KAT myc-Tip60 acetylates SBP-mycFe65 in HEK293 cells (Figure 2B). Quantification showed that co-expression of the Tip60 mutant abolished the Fe65 acetylation signal and also resulted in lower levels of coeluted Tip60 (Figure 2C, D). $\triangle$ KAT Tip60 is a double mutant and we found that already the single mutations Q377E and G380E reveal the same reduction of acetylation levels for Fe65 and Tip60, as well as reduced pulldown of Tip60 by Fe65 (Figure S2B). 


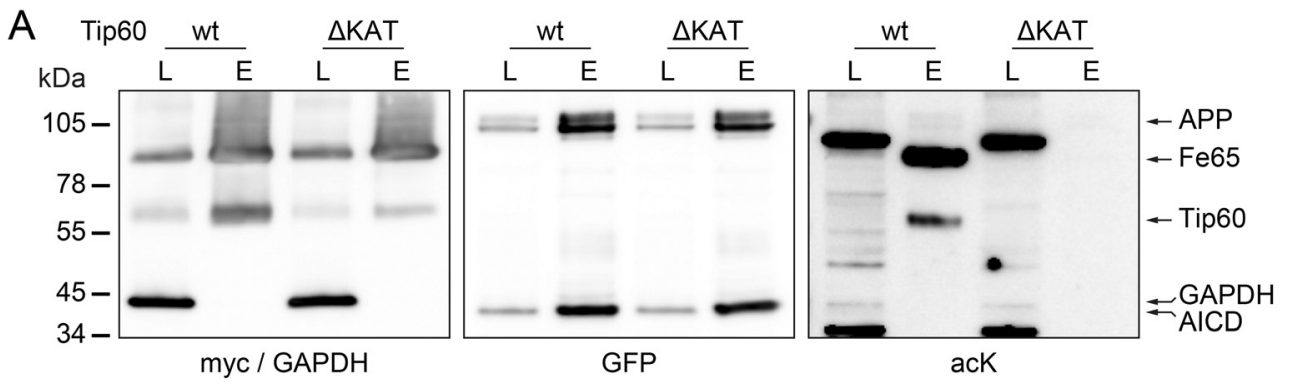

B

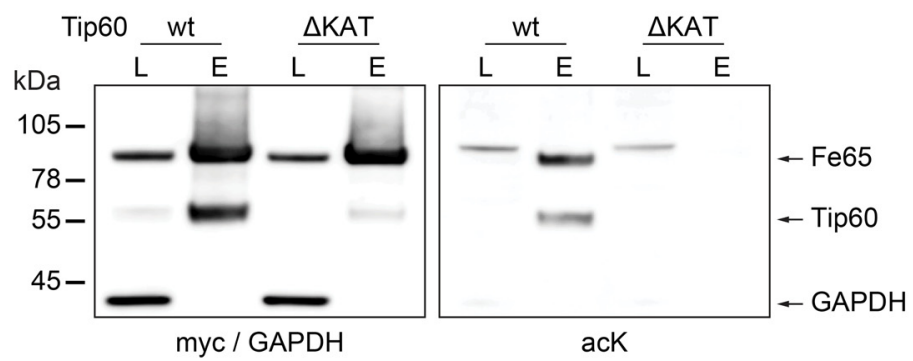

$E$
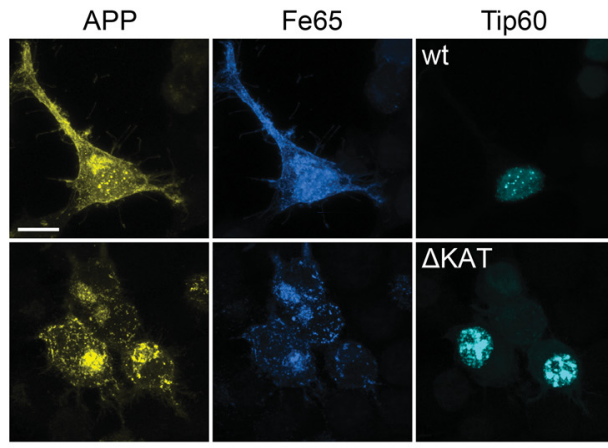

overlay/DAPI

C
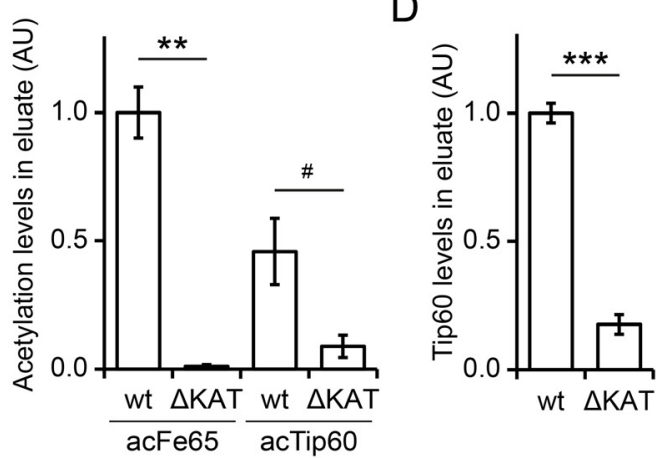

$\mathrm{F}$

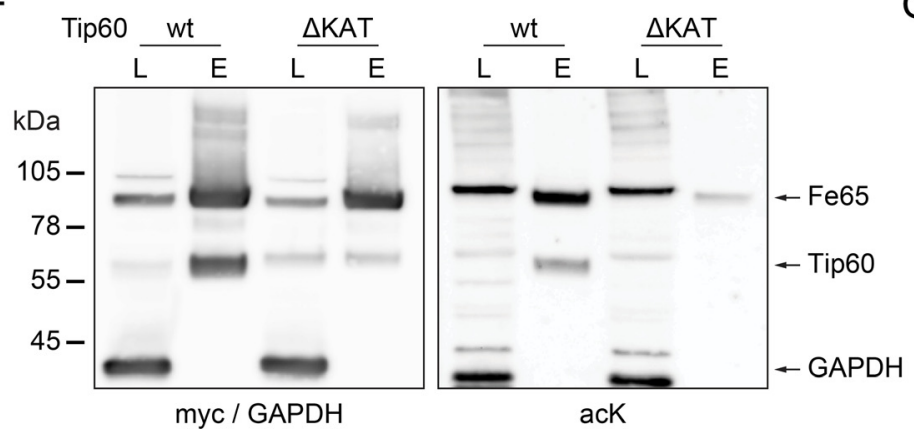

G

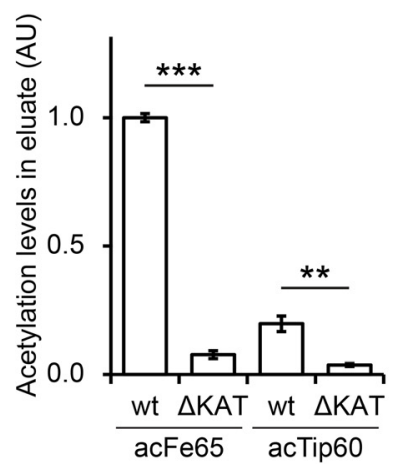

$\mathrm{H}$

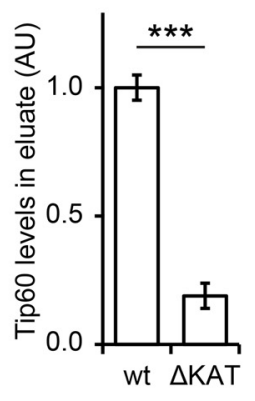

Figure 2: Tip60 KAT activity autoacetylates Tip60 and acetylates Fe65.

(A-D) Dynabeads-Streptavidin-based pulldown of SBP-myc-Fe65 from transfected HEK293 cells and subsequent Western blot analysis of lysates (L) and eluates (E). (A) Co-expression of SBP-myc-Fe65 with APP-Cit and wt or $\triangle K A T$ myc-Tip60. Fe65 pull down co-elutes APP, AICD and Tip60, but only Fe65 and Tip60 are acetylated with expression of wt Tip60. (B) Co-expression of SBP-myc-Fe65 and myc-Tip60 without APP. Pulldown of Fe65 co-isolates Tip60 and both are acetylated by wt, not by $\triangle K A T T i p 60$. (C and D) Quantification of eluates determining the ratio of acetylated Fe65 vs. Fe65, acetylated Tip60 vs. Tip60 and the amount of co-precipitated Tip60 (mean \pm SEM, $n=3$; two-tailed $t$-test). (E-H) Analysis of AFT spot formation, Tip60 autoacetylation and Fe65 acetylation in N2a cells. (E) Co-expression of APP-Cit and HA-Fe65 with wt CFP-Tip60 (top row) leads to AFT spot formation, while the $\triangle$ KAT CFP-Tip60 (bottom row) is retained in speckles. Scale bar: $10 \mu \mathrm{m}$. (F-H) Co-expression of SBP-myc-Fe65 and wt or $\triangle$ KAT myc-Tip60 followed by Streptavidin-based pulldown in N2a cells. (F) Representative Western blots of lysates (L) and eluates (E). Wt Tip60 acetylates Fe65 and Tip60 whereas the Tip60 $\triangle$ KAT mutant does not. ( $\mathrm{G}$ and H) Quantification of eluates determining the ratio of acetylated Fe65 vs. Fe65, acetylated Tip60 vs. Tip60 and the amount of co-precipitated Tip60 (mean \pm SEM, $n=3$ for (G) and $n=4$ for (H); two-tailed $t$-test). 
We repeated the experiments in mouse N2a cells. Confocal microscopy showed nuclear AFT spot formation when co-expressing wt Tip60, but the $\triangle \mathrm{KAT}$ mutant again abolished spot formation and Tip60 resided in speckles (Figure 2E). Pulldown of SBP-myc-Fe65 and Western blot analysis again revealed acetylation of Fe65 and autoacetylation of Tip60 (Figure 2F). Quantification showed strong reduction in Fe65 acetylation levels and Tip60 autoacetylation when $\triangle$ KAT instead of wt Tip60 is coexpressed (Figure $2 \mathrm{G}$ ) and less interaction of the Tip60 mutant with Fe65 (Figure 2H). In conclusion, these data show that the Tip60 KAT activity is required for Tip60 autoacetylation and the acetylation of Fe65.

\section{Fe65L1 and Fe65L2 are not acetylated by Tip60}

Fe65 belongs to a protein family that contains two further homologs, Fe65L1 and Fe65L2. We have shown that AFT spot formation and transcriptional activity is a unique property of Fe65 (Probst et al. 2020). We co-expressed SBP-myc-tagged Fe65L1 and Fe65L2 constructs with wt Tip60 in HEK293 cells. Fe65L1 did not co-precipitate Tip60 and showed only minor acetylation signals when compared to Fe65 that was tested in parallel (Figure 3A). Fe65L2 is the smallest family member and as its molecular weight is comparable to myc-Tip60, we used co-transfection of CFP-Tip60 to differentiate the two proteins. In parallel experiments using CFP-Tip60 we again clearly detect acetylation of Fe65 (Figure 3B). In contrast, Fe65L2 shows no acetylation signal and does not co-precipitate Tip60. We conclude that Fe65 is the sole member of the Fe65 protein family that interacts with Tip60 and gets acetylated by its KAT activity.

\section{Tip60 acetylates Fe65 K204 and K701}

To identify which lysine residues in Fe65 (Figure S3) are acetylated by Tip60, we performed mass spectrometry analysis. Liquid chromatography electrospray ionization tandem mass-spectrometry (LC/ESI-MS/MS) and matrix-assisted laser desorption/ionization tandem massspectrometry (MALDI-MS/MS) analyses revealed multiple acetylated lysine residues in SBP-myc-Fe65 when coexpressed with wt myc-Tip60. While most of those acetylated lysine residues could not be consistently detected throughout different biological replicates and technical approaches, K204 and K701 were identified multiple times to be acetylated (Figure 4A and Figure S4A, B).
We mutated the identified lysine residues to arginine, a mimic of non-acetylated lysine that retains the positive charge, and performed pulldown and Western blot experiments. Indeed, mutation of either lysine 204 or 701 to arginine, reduced the acetylation level of SBP-myc-Fe65 (Figure 4B, C). Simultaneous mutation of both lysine residues to arginine (K204/701R) prevented acetylation of SBP-myc-Fe65. Additional mutation of the remaining 23 lysine residues in Fe65 and of the two lysine residues in the SBP- and myc-tags to arginine (all KtoR) similarly abolished the acetyl-lysine signal. Tip60 binding to the Fe65 mutant proteins was not significantly altered by the mutations, except for a reduced binding of the all KtoR mutant (Figure 4D). Finally, mutation of all lysine residues in SBP-myc-Fe65, except of Fe65 lysine residues 204 and 701, enabled acetylation of SBP-myc-Fe65 (Figure 4B, C). This suggests that K204 and K701 are the major lysine residues in Fe65 that are acetylated by Tip60. The residual minor acetyl-signal of the K204/701R and all KtoR mutants could originate from co-precipitated endogenous Fe65, as Fe65 has been reported to form dimers (Feilen et al. 2017).

\section{Acetylation of Fe65 increases its transcriptional activity but is not necessary for AFT spot formation}

We analyzed whether preventing the charge neutralization induced by acetylation of K204 and K701 would influence AFT spot formation. Neither single K204R or K701R mutation (Figure S5A), nor the double mutation (Figure 4E) or the all KtoR mutation (Figure S5A) prevented AFT spot formation. The $\mathrm{K}$ to $\mathrm{R}$ mutation preserves the positive charge, mimicking the positive charge of a non-acetylated lysine. We also exchanged the two lysine residues to glutamine, thereby eliminating the positive charge, a major outcome of acetylation. When these mutants were cotransfected with APP-Cit and CFP-Tip60, both the single K to Q mutants (Figure S5A) and the K204/701Q double mutant (Figure 4E) were able to support AFT spot formation. This suggests that while AFT spot formation is only possible with a functional Tip60 KAT, Fe65 acetylation itself is not necessary.

We further analyzed if mutation of lysine residues 204 and 701 affects the dimerization capacity of Fe65 in pulldown experiments. Lysine 204 is located in the linker 1 region preceding the WW domain and lysine 701 in the C-terminus following the PTB2 domain. In contrast to deletion of the PTB2 domain that disrupts Fe65 dimerization (Feilen et al. 2017; Probst et al. 2020), neither the 


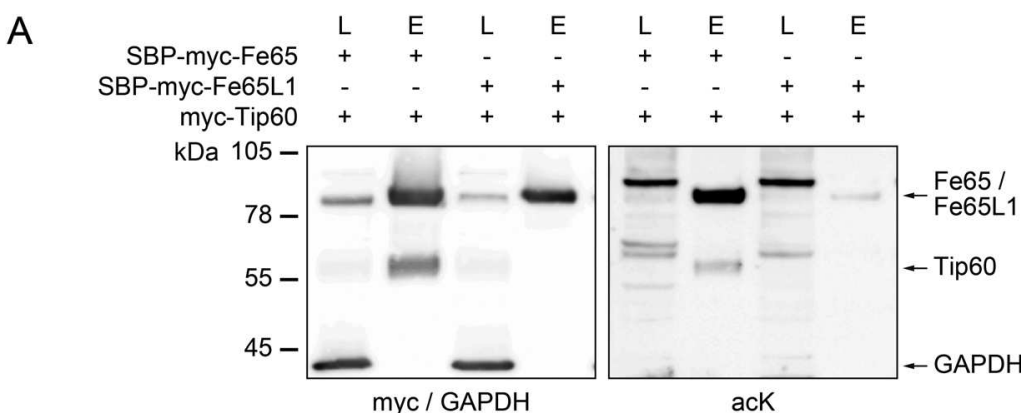

B

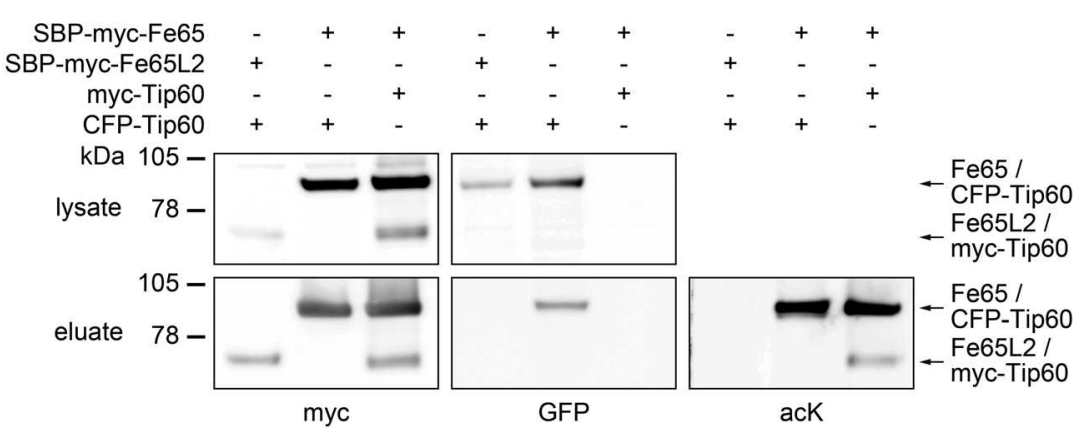

Figure 3: Fe65L1 and Fe65L2 are not acetylated by Tip 60 .

Co-expression of SBP-tagged Fe65 family members with wt myc-Tip60 or CFP-Tip60 in HEK293 cells followed by Streptavidinbased pulldown and Western blotting of lysates (L) and eluates (E). (A) Tip60 does not interact with or acetylate Fe65L1, while it acetylates Fe65 tested in parallel. (B) Tip60 does not interact with or acetylate Fe65L2. Because Fe65L2 runs at the same height as myc-Tip60, the larger CFP-Tip60 was used. Fe65 tested in parallel interacted with and was acetylated by both Tip60 constructs.
K204/701R nor the K204/701Q mutations affected dimerization (Figure S5B).

To analyze the effect of acetylation on transcriptional activity, we used our recently described transcription assay (Konietzko et al. 2019). We designed Gal4-DBD-fused Fe65 constructs harboring $\mathrm{K}$ to $\mathrm{R}$ or $\mathrm{K}$ to $\mathrm{Q}$ mutations and transfected these into HEK2:UAS-Cit-NLS reporter cells. Nuclear Cit expression was quantified in confocal microscopy images and normalized to the number of DAPI-stained nuclei (Figure 4F). The K204R and K701R single mutants did not have an effect, but the K204/701R double mutant slightly but significantly reduced Fe65 transcriptional activity. In contrast, the acetylation-mimicking mutant K701Q, and even stronger the K204/701Q double mutant, increased transcriptional activity. These data suggest that acetylation of K204 and K701 increases Fe65 transcriptional activity.

\section{Effect of KATs and KDACs on Fe65 acetylation levels and nuclear spot formation}

We assessed whether the KDAC Sirt1 reduces Fe65 acetylation levels. We co-expressed SBP-myc-Fe65 and myc-Tip60 together with wt N-terminally FLAG-tagged Sirt1 or with the catalytically inactive version (H363Y) in HEK293 cells. Pulldown of Fe65 was followed by Western blot analysis (Figure 5A). Quantification revealed that
Sirt1 reduces the acetylation levels of Fe65, but not significantly of Tip60 (Figure 5B). Moreover, the amount of coprecipitated Tip60 was not affected by wt Sirt1 as compared to the inactive H363Y mutant (Figure 5C).

We analyzed whether Fe65 can be acetylated by other acetyltransferases. Tip60 is a member of the MYST family of acetyltransferases and its closest homolog is hMOF (Neal et al. 2000). HEK293 cells were co-transfected with SBP-myc-Fe65 and either wt or $\triangle$ KAT myc-Tip60 or hMOF-myc. Pulldown and Western blot analysis showed that only wt Tip60 acetylates Fe65, whereas hMOF expression does not lead to Fe65 acetylation (Figure 5D). Both, Tip60 and hMOF are able to acetylate the K120 of p53 (Sykes et al. 2006). To verify that lack of Fe65 acetylation by hMOF is not due to lack of KAT activity by the construct, we cloned SBP-myc-p53 as a positive control substrate. Indeed, co-expression of hMOF-myc and wt myc-Tip60 but not of $\triangle$ KAT myc-Tip60 caused acetylation of SPB-myc-p53 at K120 (Figure 5D). Confocal microscopy analysis revealed a homogenous nuclear distribution of hMOF, as opposed to the speckles seen with Tip60 (Figure 5E, upper row). Co-transfection of hMOF with Fe65 did not result in relocation of $\mathrm{Fe} 65$ to specific nuclear sites as seen for Tip60 (Figure 5E, lower row). We used Cerulean-tagged Fe65 (Cer-Fe65), because antibody-staining is often occluded in the nuclear environment.

We analyzed members from the other two major KAT families. HEK293 cells were co-transfected with SBP-myc-Fe65 and Cit-HA as a control, or the KATs Tip60, 
A

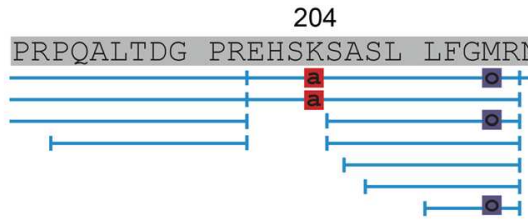

$\mathrm{B}$
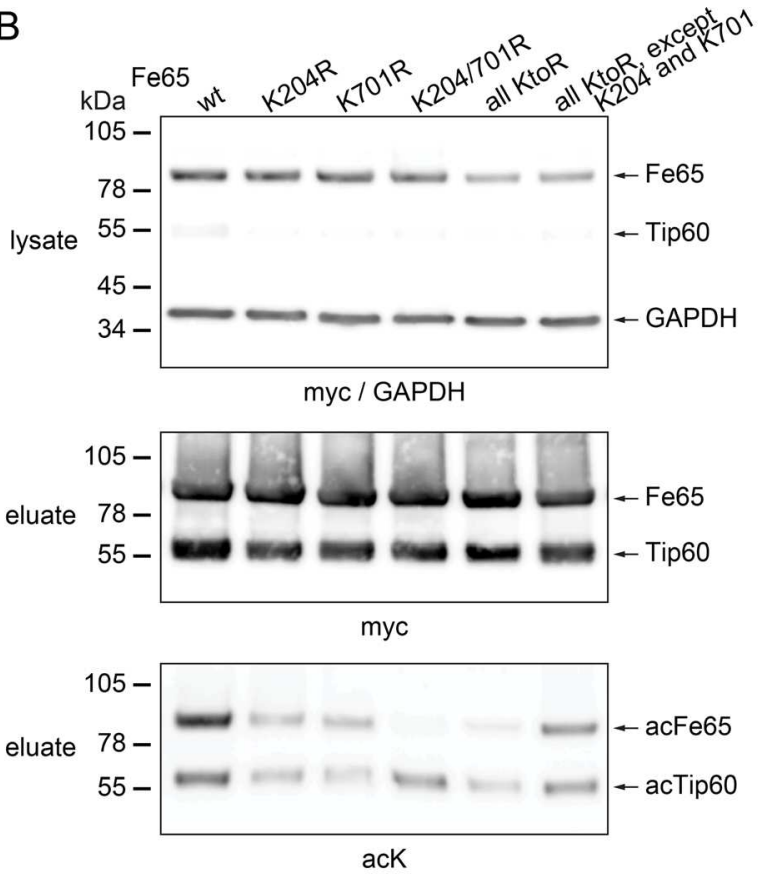

$E$

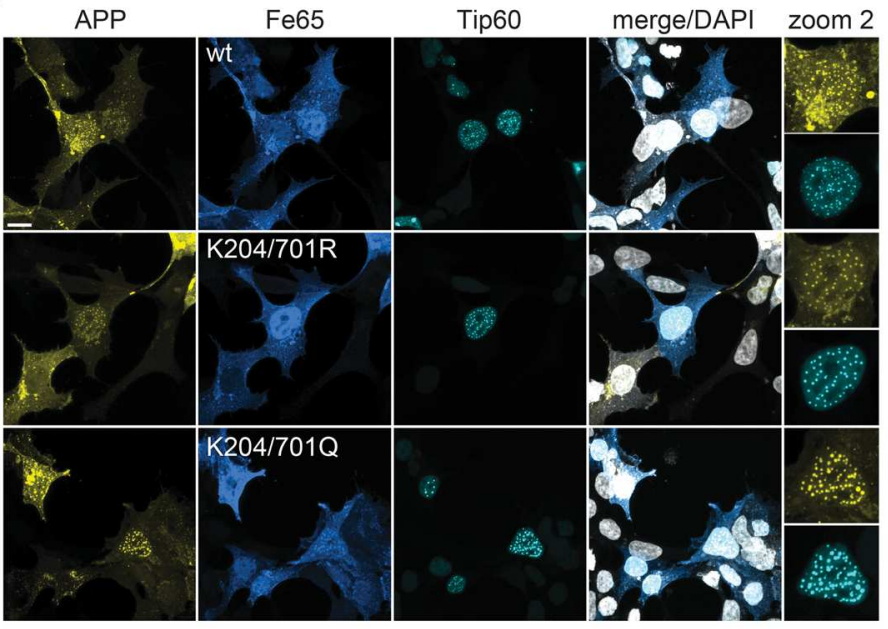

701

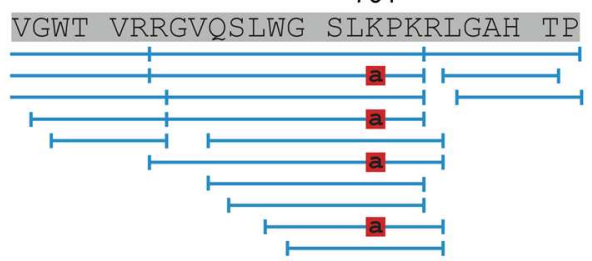

C

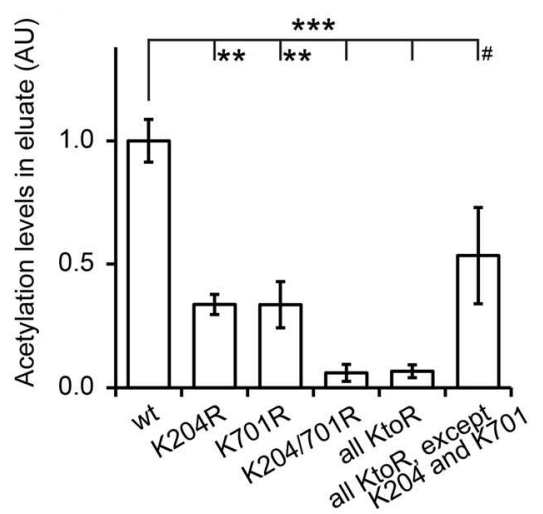

D

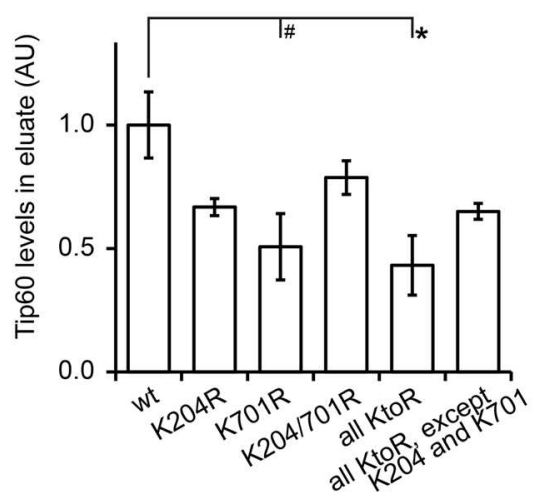

$\mathrm{F}$

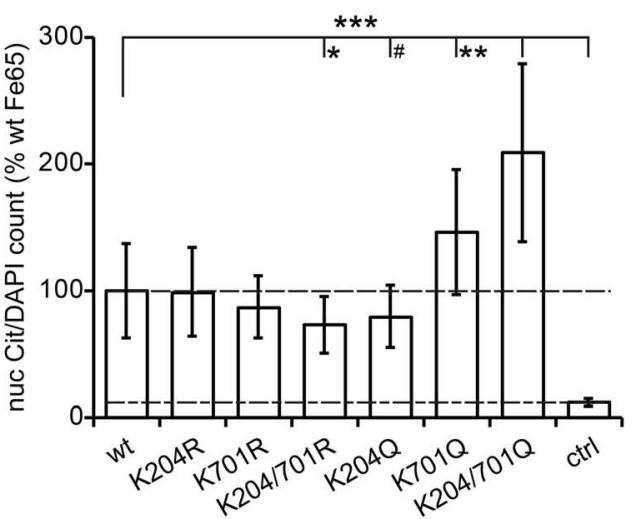

Figure 4: Tip60 acetylates Fe65 K204 and K701-an acetylation-mimicking Fe65 mutant reveals increased transcriptional activity.

(A) The LC/ESI-MS/MS repeatedly identifies K701 and K204 of SBP-myc-Fe65 being acetylated (red squares), when co-expressed with wt mycTip60 in HEK293 cells. Oxidized methionine (purple squares) is also marked as it influences peptide identification. (B-D) Expression of wt Tip60 together with wt SBP-myc-Fe65, or lysine to arginine mutants thereof, in HEK293 cells, followed by pulldown and Western blot analysis. (B) Representative western blots of lysates and eluates. (C) Quantification of eluates showing the ratio of acetylated Fe65 vs. Fe65. (D) Quantification of co- precipitated Tip60 shows similar amounts for most constructs. Mean \pm SEM of $n=3$ are shown for (C) and (D) (two-tailed $t$ test). (E) Confocal microscopy of HEK293 cells co-transfected with APP-Cit, CFP-Tip60 and SBP-myc-Fe65 with wt sequence or K204 and K701 mutated to either arginines or glutamines. All Fe65 mutants are able to form AFT spots. Scale bar: $10 \mu \mathrm{m}$. (F) UAS-Cit assay in HEK2:UAS-Cit-NLS cells to analyze the transcriptional activity of different Gal4-myc-Fe65 mutants. Mutation K701 or of K204 together with K701 to glutamine significantly enhanced the transcriptional activity of Fe65. Non-transfected cells were used to measure baseline Cit fluorescence (ctrl). Mean \pm SD of $n=21$ images from 3 replicates are shown (Mann-Whitney $U$ test). 
A

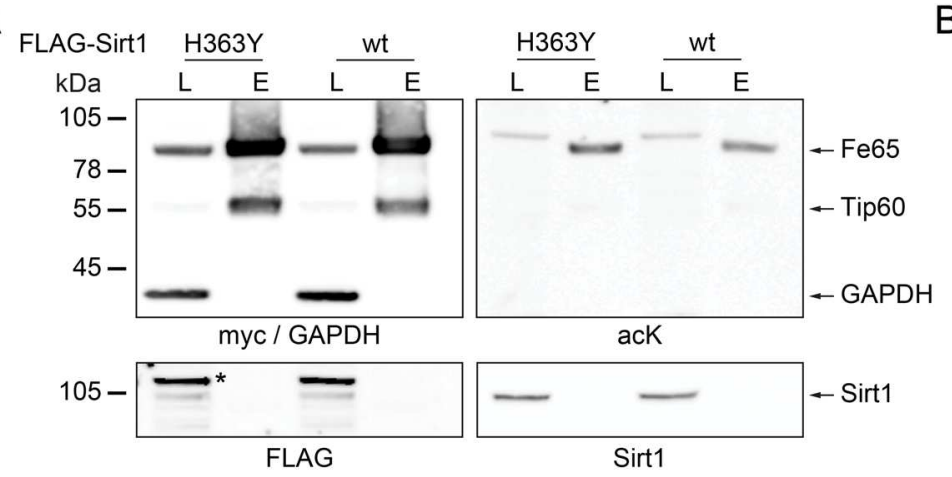

D

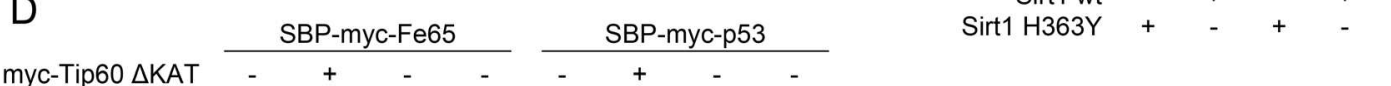

B

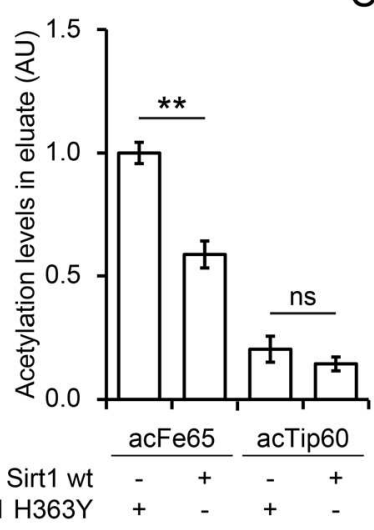

C

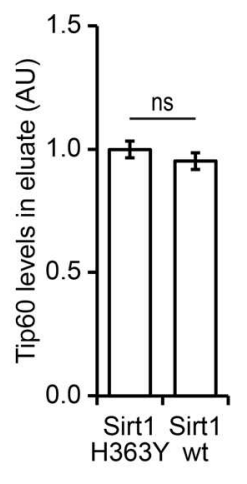

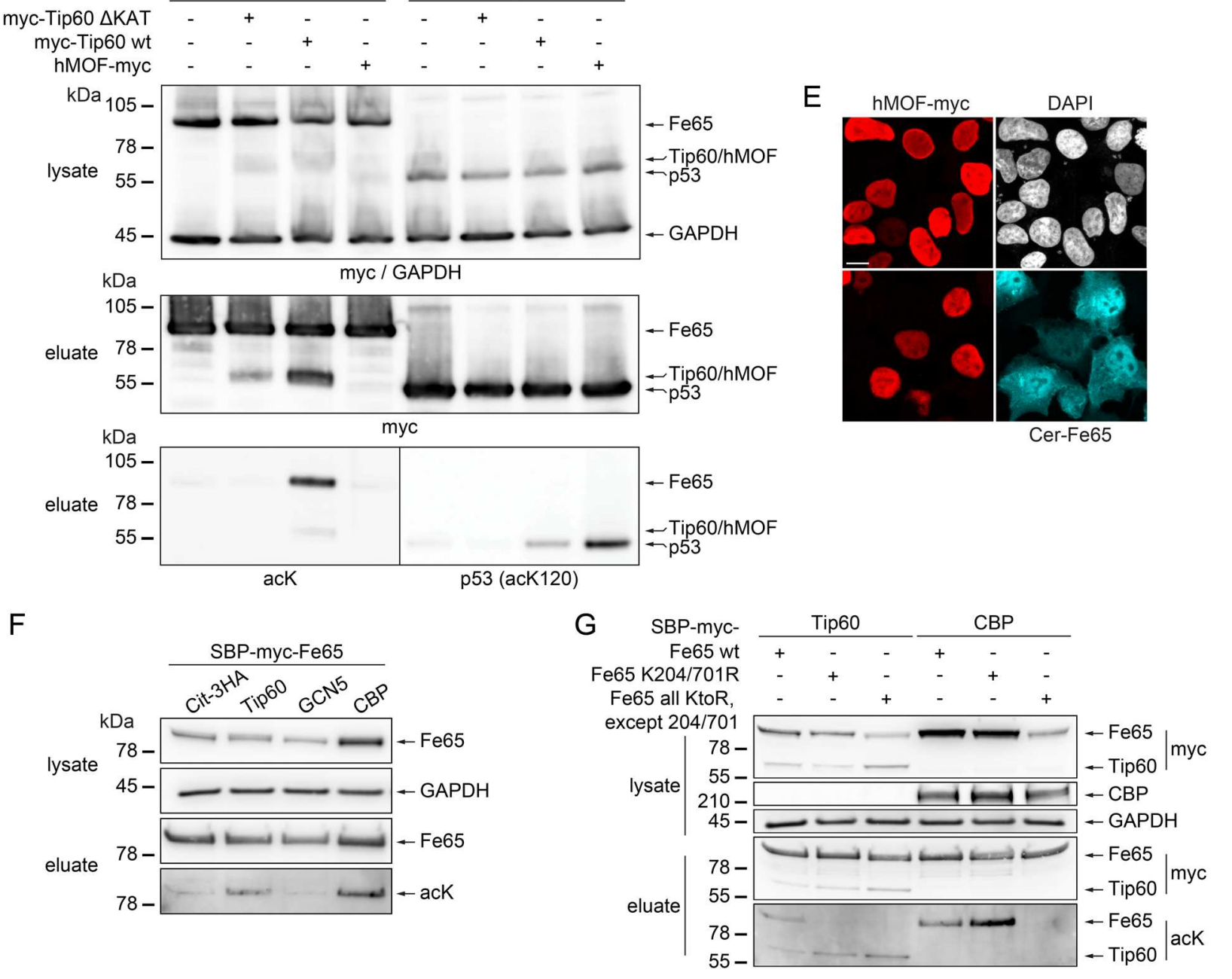

Figure 5: Effect of Sirt1 and different KATs on Fe65 acetylation levels.

(A-C) Dynabeads-Streptavidin-based pulldown of SBP-myc-Fe65 from HEK293 cells co-transfected with myc-Tip60 and either wt or mutant (H363Y) FLAG-Sirt1, followed by subsequent Western blotting of lysates (L) and eluates (E). (A) Representative western blots. Band marked by a star is unspecific staining by the anti-FLAG antibody. (B) Quantification of eluates showing the ratio of acetylated Fe 65 vs. Fe 65 and acetylated Tip60 vs. Tip60. (C) Quantification of co- precipitated Tip60. Mean \pm SEM of $n=4$ are shown for (B) and (C) (two-tailed $t$-test). (D) SBP-myc-Fe65 or SBP-myc-p53 were co-expressed with wt myc-Tip60, KAT myc-Tip60 or wt hMOF-myc in HEK293 cells, followed by pulldown and Western blot analysis. In contrast to wt Tip60, its closest homolog hMOF does not acetylate Fe65. Acetylation of SBP-myc-p53 through wt myc-Tip60 and hMOF-myc confirms the activity of hMOF-myc. (E) Confocal microscopy images of hMOF-myc show a uniform nuclear localization (upper row). Co-transfection of Cer-Fe65 with hMOF-myc did not result in Fe65 localizing to specific nuclear structures (lower row). Scale bar: $10 \mu \mathrm{m}$. (F) SBP-myc-Fe65 was co-expressed with Cit-3HA, myc-Tip60, GFP-GCN5 or CBP-HA in HEK293 cells, followed by pulldown and Western blot 
GCN5 or CBP. Pulldown and Western blot analysis revealed acetylation of Fe 65 by Tip 60 and CBP, not by GCN5 (Figure 5F). In addition, Fe65 levels in the lysate were strongly increased with CBP co-expression. To study if CBP targets the same lysine residues as Tip60, we repeated the pulldown experiments with the Fe65 K204/701R mutant and the all KtoR except K204/701 mutant (Figure 5G). The latter mutant showed reduced expression levels as also seen in Figure 4B. Nevertheless, pulldown leads to isolation of similar Fe65 levels. Whereas the acetylation signal induced by Tip60 is lost in the K204/701R mutant, CBP acetylates it to similar levels as wt Fe65. The acetylation signal induced by CBP is lost in the Fe65 all KtoR except K204/701 mutant. Again, Fe65 levels increased when CBP was co-expressed, but not for the all KtoR except K204/701 mutant. Quantification of $n=7$ separate transfections of Tip60 or CBP, normalized to GAPDH, revealed 3-5-fold higher Fe65 levels with CBP. The data show that Tip60 and CBP can acetylate Fe65 at different lysine residues.

Confocal microscopy of transfected HEK293 cells showed that CBP is mainly localized to the nucleus, where it is often detected in punctate structures (Figure 6A, upper row). Nevertheless, co-transfection of Cer-Fe65 with CBP did not result in Fe65 relocation to nuclear spot structures (Figure 6A, lower row). Co-transfection of myc-Fe65 with CFP-Tip60 reliably induces spot formation that can be clearly observed via the fused fluorescent protein, less so with antibodymediated staining the myc tag. Co-transfection of Tip60, Fe65 and CBP resulted in spot formation that also attracted CBP. The staining of ring-like structures surrounding spots is frequently observed and relates to the inability of antibodies to access the center of nuclear spots (Figure 6B).

\section{Effect of KATs and KDACs on Fe65 transcriptional activity}

To further elucidate the impact of acetylation on Fe65 transcriptional activity we analyzed the effects of pharmacological treatments. HEK2:UAS-Cit-NLS reporter cells were transfected with Gal4-Fe65 alone or together with Tip60 (Figure 6C, D). Nicotinamide (NAM), an inhibitor of the sirtuin family of KDACs, did not increase Fe65 transcriptional activity both, with and without co-expression of Tip60. Resveratrol, an activator of the $\mathrm{NAD}^{+}$-dependent class III KDAC Sirt1, caused a significant reduction of Fe65 transcriptional activity. Coexpression of the KAT Tip60 counteracted the effect of resveratrol. Fe65 transcriptional activity was enhanced with the selective class I/II KDAC inhibitor Trichostatin A (TSA), but only when Tip60 was co-expressed. TH1834, a Tip60-selective KAT inhibitor (Gao et al. 2014), decreased the transcriptional activity of Fe65 in a dose-dependent manner, irrespective of the co-expression of Tip60. These experiments were performed in the saturated range of the assay, using lipofection of $1 \mu \mathrm{g}$ of DNA. We have reported that modulation of APP-Fe65 signaling by mutation of phosphorylation sites could only be detected in the linear range of the assay, whereas a mutation abolishing APP-Fe65 interaction showed a similar reduction in the saturated range (Konietzko et al. 2019).

We therefore repeated the assay in the linear range (150 ng DNA), to better identify modulatory changes in both directions. We report increased transcriptional activity by inhibition of type I/II KDACs with TSA or by inhibition of type III KDACs with NAM (Figure 6E). Similar to its effects in the saturated range, inhibition of Tip60 with TH1834 strongly decreased activity. In addition to measuring nuclear Cit intensity as the readout of transcriptional activity, we also stained the Gal4-Fe65 constructs via the myc-tag. As Fe65 is localized throughout the cell, we measured total fluorescence levels and normalized them to the number of DAPI-stained nuclei (Figure 6F). Neither NAM treatment nor TH1834 influenced expression levels of Gal4-Fe65. In contrast, TSA treatment at both concentrations resulted in around two-fold higher Fe65 levels. This could explain why no effect on transcription was observed with TSA at saturating DNA concentrations-because protein levels are already saturated, further increase does not lead to a higher transcriptional readout. In contrast, TH1834 inhibits transcriptional activity in both saturating and linear conditions. Inhibition of acetylation by Tip60 affects all expressed Gal4-Fe65, irrespective of cellular levels. Transcriptional activity of Fe65 was further increased when TSA and NAM were combined, but addition of NAM did not increase Fe65 levels over the increase caused by TSA alone. Thus, both Tip60 and class III KDACs modulate the transcriptional activity of Fe65 without influencing protein levels.

To further elucidate the effect of Tip60 on Fe65mediated transcription, we analyzed wt Gal4-Fe65 and

analysis. In addition to Tip60, CBP also acetylates Fe65, whereas GCN5 expression did not increase acetylation signals. (G) Pulldown and analysis of acetylation levels revealed that CBP does not acetylate K204/701. Whereas the acetylation of Fe65 by Tip60 is strongly reduced in the K204/701R mutant, this mutant can be acetylated by CBP. The all KtoR, except K204/701 mutant Fe65 was not acetylated by CBP. Coprecipitation of Tip60 is observed with all Fe65 species (eluate, myc blot), but no CBP was identified in the eluates of the pulldowns (not shown). Fe65 levels are consistently increased when CBP is co-expressed (F, G). 
A

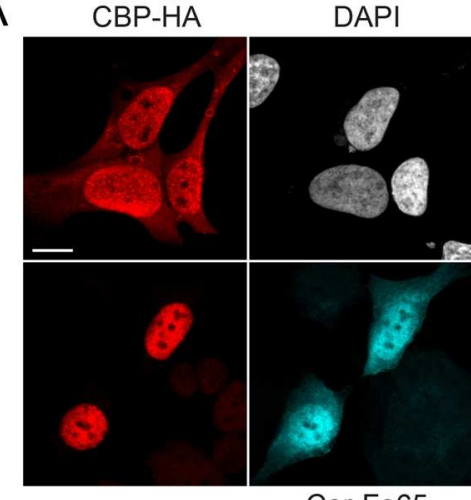

Cer-Fe65

C

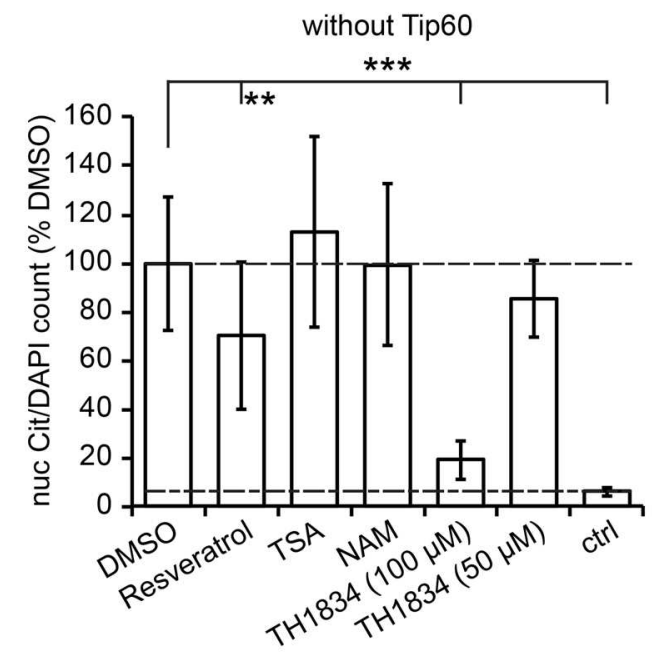

$E$

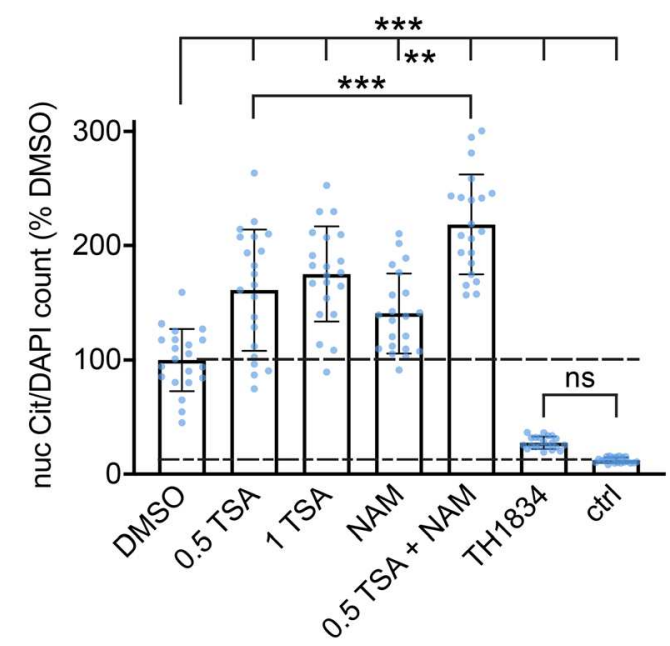

B

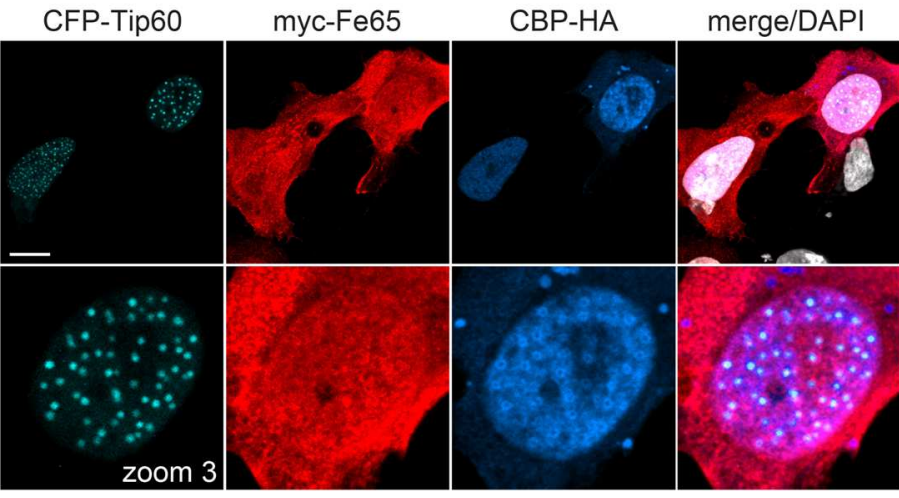

D

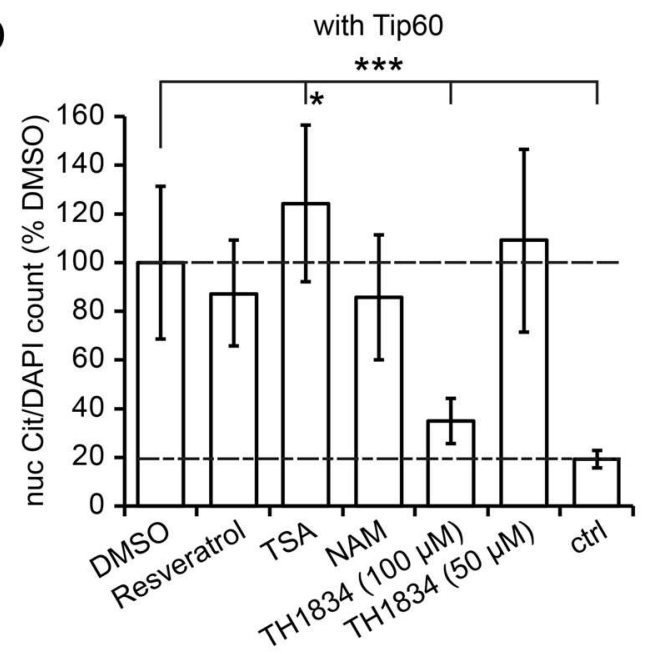

$\mathrm{F}$

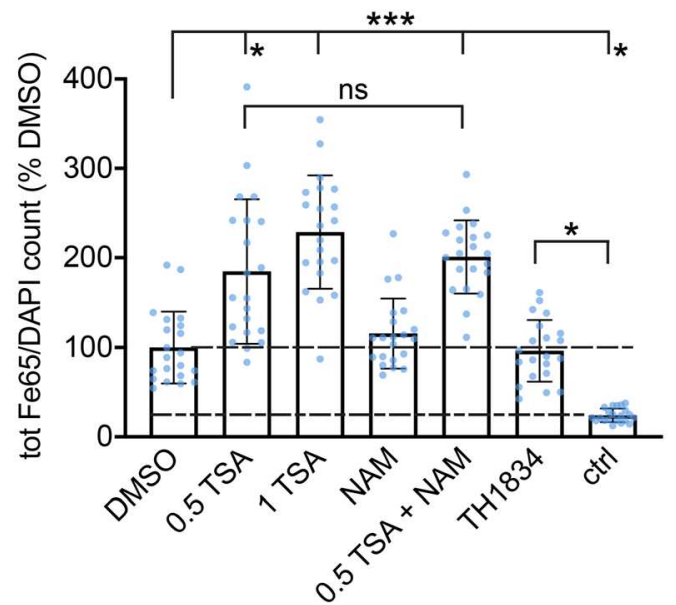

Figure 6: Effect of KATs and KDACs on Fe65 transcriptional activity.

(A) Confocal microscopy of HEK293 cells expressing CBP-HA alone (upper row), or together with Cer-Fe65 (lower row). (B) Co-expression of CFP-Tip60 and myc-Fe65 leads to the formation of nuclear spots that also attract CBP. Spots are clearly identified via the fused fluorescent protein, whereas the myc antibody does not properly stain these structures. HA-tag staining for CBP presents as ring structures, due to inaccessibility of the center of spherical spots for antibodies. Scale bars: $10 \mu \mathrm{m}$. (C and D) UAS-Cit assay in HEK2:UAS-Cit-NLS cells transfected with Gal4-myc-Fe65 with or without Tip60 $(1 \mu \mathrm{g}$ each) and treated with the indicated pharmacological agents. Mock treatment of transfected cells was done with DMSO and non-transfected cells were used to measure baseline Cit fluorescence (ctrl) (mean \pm SD of $n=21$ images from 3 replicates; one-way ANOVA with Bonferroni correction). Drug concentrations are: $100 \mathrm{nM}$ Resveratrol, $500 \mathrm{nM}$ Trichostatin A 
the two K204/701 mutants in the UAS-Cit assay, with coexpression of wt Tip60, $\triangle$ KAT Tip60 or CFP-NLS as a control (Figure 7A). The Fe65 K204/701Q mutant again significantly increased transcriptional activity. The coexpression of wt Tip60 reduced transcriptional activity of all three Fe65 constructs, while retaining the enhanced transcription of the K204/701Q mutant compared to wt and the K204/701R mutant. In contrast, the $\Delta$ KAT Tip60 did not influence transcription, compared to co-expression of CFP-NLS. In conclusion, while the acetylation-mimicking K204/701Q Fe65 mutant increases transcriptional activity, the co-expression of Tip60 that acetylates these residues leads to a reduction in activity in the UAS-Cit assay.

\section{Tip60 autoacetylation is required for AFT spot formation and Tip60 stabilization}

Although acetylation of Fe65 by Tip60 has an effect on transcriptional activity, it is not essential for AFT spot formation. Thus, lack of Fe65 acetylation cannot explain the localization of $\triangle$ KAT Tip60 to speckles and the inability to form AFT spots. We tested if autoacetylation might be required for AFT spot formation. For this, we co-expressed differently tagged wt and $\triangle$ KAT Tip60 constructs together with Fe65. While both mycand CFP-tagged wt Tip60 form spots together with Fe65, the $\triangle \mathrm{KAT}$ mutants were retained in speckles (Figure $7 \mathrm{~B}$ ). As reported, detection of nuclear structures by fluorescent proteins is superior to antibody-mediated staining due to the crowded nuclear environment. We therefore combined Fe65 with the wt myc-tagged Tip60 and the $\triangle$ KAT CFP-tagged Tip60. In this condition, we clearly detected the localization of the $\triangle \mathrm{KAT}$ CFP-Tip60 to nuclear spots (Figure 7B).

With pulldown of SBP-tagged Fe65 we had observed a clear reduction in the amount of co-precipitated Tip60 when comparing the $\triangle \mathrm{KAT}$ with the wt (Figure 2D,H). To gather more insight into the role of Tip60 autoacetylation, we determined the protein half-life of the Tip60 variants. HEK293 cells were transfected with wt or $\triangle$ KAT Tip60 with or without co-expression of Fe65 and treated for varying time points with the protein synthesis inhibitor cycloheximide (CHX) (Figure 7C). Western blots of Fe65 expression and endogenous GAPDH levels are shown in Figure S6.
Quantification of protein levels in Western blots revealed a similar half-life of around $2 \mathrm{~h}$ for both, wt and $\triangle$ KAT Tip60 (Figure 7D). Co-expression of Fe65 resulted in a more than doubling of the half-life of wt Tip60, whereas for the $\triangle$ KAT mutant we detected a minor decrease in half-life.

\section{Discussion}

Here, we reveal the crucial role of the KAT Tip60 for nuclear signaling by APP/Fe65. Autoacetylation of Tip60 is essential for the dissolvement of nuclear speckles and the formation of AFT spots. The acetylation of Fe65 by Tip60 increases its transcriptional activity. Tip60 acetylates many nuclear proteins and functions in DNA repair, apoptosis and transcription (Sapountzi et al. 2006). We mutated the amino acids reported to disrupt the KAT activity of Tip60 (Ikura et al. 2000) and analyzed the effects on nuclear signaling by APP/Fe65.

The majority of Tip60 is detected in nuclear speckles. When Fe65 is co-expressed there is a dramatic relocation of Tip60 and Fe65 to spherical spots that represent sites of transcription (Konietzko et al. 2010; von Rotz et al. 2004). In contrast, $\triangle$ KAT Tip60 does not relocate to spots with Fe65 but both co-localize in nuclear speckles. Identical results were seen in HEK293 and N2a cells, when using confocal microscopy of fluorescent protein-tagged constructs or flow cytometry of BiFC using split YFP. Interaction of Fe65 with $\triangle$ KAT Tip60 is reduced, as seen with reduced coprecipitation in pulldown experiments. Also, when APP is co-expressed, the majority of Fe65 is retained in the cytoplasm by APP and no co-localization with Tip60 in speckles is detected. As we have discussed in a recent paper (Probst et al. 2020), reduced interaction can still enable colocalization in situ but disrupts pulldown, probably due to the harsh washing conditions without prior fixation. The KAT activity of Tip60 is therefore necessary for proper interaction with Fe65, enabling the relocation of both to nuclear spots. With immunoprecipitation experiments it has been reported that Tip60 KAT activity is necessary for the formation of the trimeric AFT complex (Baek et al. 2002), which is in line with our pulldown data. In a recent paper, we show that Tip60 and Fe65 interact in the

(TSA), $5 \mathrm{mM}$ nicontinamide (NAM) and $50 \mu \mathrm{M}$ or $100 \mu \mathrm{M}$ TH1834. (E and F) UAS-Cit assay performed in HEK2:UAS-Cit-NLS cells in the linear range with transfection of $150 \mathrm{ng}$ Gal4-myc-Fe65. Drug treatments were $500 \mathrm{nM}$ and $1 \mu \mathrm{M}$ ( 0.5 and 1) TSA, $5 \mathrm{mM}$ NAM, $500 \mathrm{nM}$ TSA + $5 \mathrm{mM}$ NAM and $100 \mu \mathrm{M}$ TH1834. (E) The nuclear Cit signal was quantified and normalized by the number of DAPI-stained nuclei. (F) Gal4-myc Fe65 was stained by myc antibodies and secondary antibodies labeled with Alexa647. Confocal microscopy was done sequentially, together with Cit and DAPI imaging. Total Alexa647 fluorescence was measured and normalized by the number of DAPI-stained nuclei (mean \pm SD of $n=21$ images from 3 replicates; one-way ANOVA with Tukey's multiple comparisons test for (E) and Kruskal Wallis with Dunn's multiple comparisons test for $(\mathrm{F})$ ). 
A

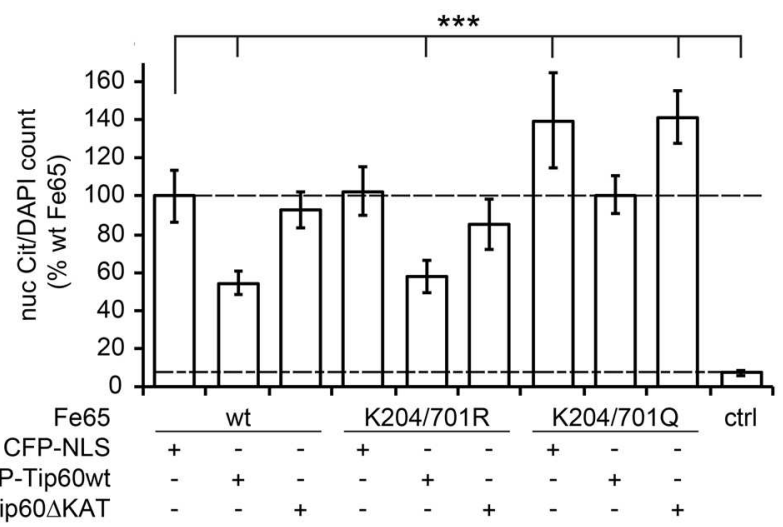

B

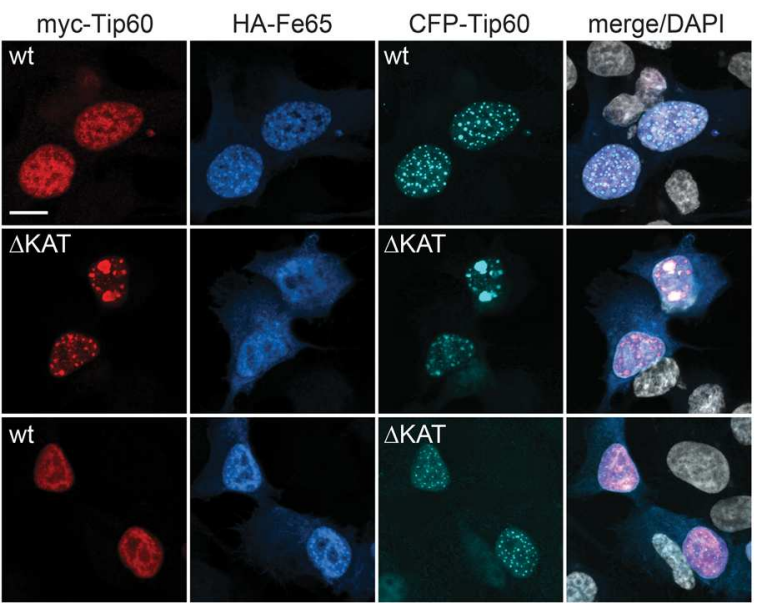

C

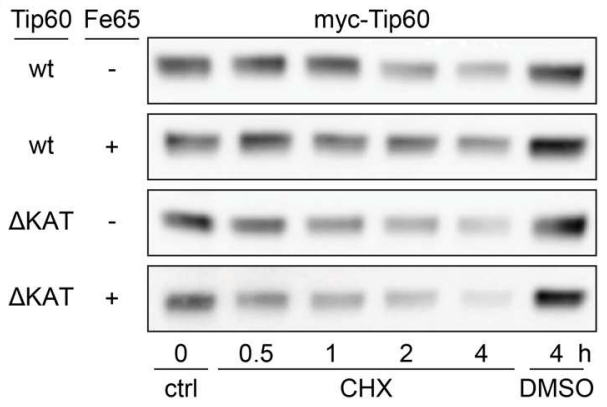

D

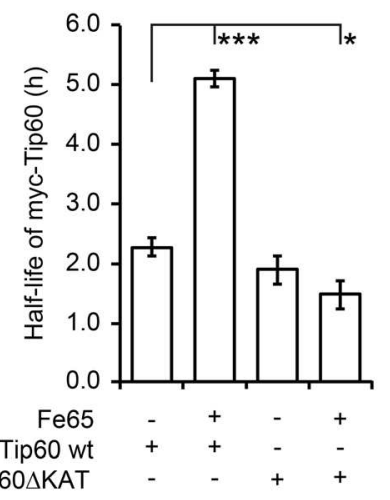

E

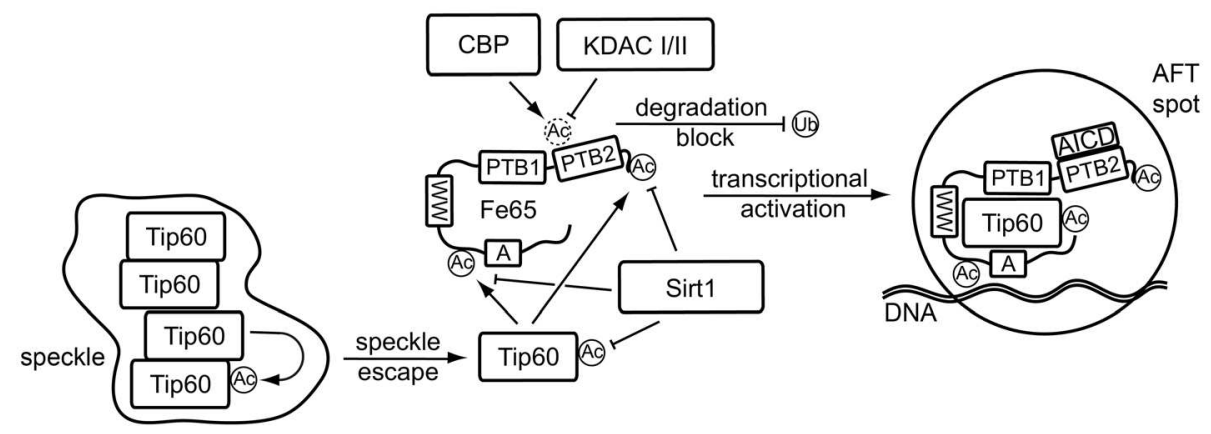

Figure 7: Role of Tip60 KAT activity in AFT spot formation and Fe65-mediated transcription.

(A) UAS-Cit assay to determine the effect of wt or $\triangle$ KAT Tip60 co-expression on the induction of Cit-NLS by Gal4-myc-Fe65 or the K204/701R and K204/701Q mutants. Co-expression of CFP-NLS was used as control (mean \pm SD of $n=21$ images from 3 replicates; one-way ANOVA with Bonferroni correction). (B) Confocal fluorescence images of HEK293 cells transfected with HA-Fe65 and both myc- and CFP-tagged Tip60. Different combinations of wt and $\triangle$ KAT Tip 60 showed formation of FT spots by $\Delta$ KAT Tip 60 when wt Tip 60 is co-expressed. Scale bar: $10 \mu \mathrm{m}$. (C and D) Protein half-life determination by CHX-mediated inhibition of protein synthesis for the indicated time points. Quantification of Western blots showed increased half-life of wt, not $\Delta$ KAT, Tip60 with co-expression of Fe65 (mean \pm SEM, $n=3$; two-tailed $t$-test). (E) Summary graphic of Fe65 regulation by acetylation/deacetylation cycles. Autoacetylation of Tip 60 is required to dissolve oligomers in nuclear speckles. Tip60 acetylates Fe65 at K204 and K701, which is counteracted by Sirt1. Acetylation by Tip60 has no effect on Fe65 turnover, but increases transcriptional activity of Fe 65 that localizes with bound Tip 60 and AICD in nuclear spots. These AFT spots are sites of gene transcription. CBP acetylates Fe65 at lysine residues different from K204 and K701, to increase the stability of Fe65. This is counteracted by class I/II KDACs. Acetylation by CBP is hypothesized to prevent ubiquitination (ubiquitin is denoted by a circled Ub). Fe65 is depicted with the acidic motif (A), the WW, PTB1 and PTB2 domains, as well as the connecting unstructured regions. Strong interaction with Tip60 involves the PTB1 domain and the N-terminal region of Fe65 (Probst et al. 2020). Acetylation is denoted by a circled Ac. In case the identity of the acetylated lysine is unknown the circle is dashed. 
cytoplasm to promote each other's nuclear translocation (Probst et al. 2020). We cannot rule out that Tip60 KAT activity is already necessary for efficient nuclear translocation. Nevertheless, $\triangle$ KAT Tip60 localizes with Fe65 to nuclear speckles but the relocation to spots is abolished, proposing that this nuclear relocation is dependent on KAT activity.

We analyzed if a component of AFT spots is acetylated by Tip60. Besides the reported autoacetylation of Tip60 (Peng et al. 2012; Wang and Chen 2010; Xiao et al. 2014; Yang et al. 2012; Yi et al. 2014), we identified acetylation of Fe65 in HEK293 and N2a cells, but we could not detect acetylation of either APP or AICD. Fe65 belongs to a family with two other members, the Fe65-like proteins Fe65L1 and Fe65L2. We have reported that only Fe65 supports APP-mediated nuclear signaling and transcription (Probst et al. 2020). Accordingly, neither Fe65L1 or Fe65L2 were acetylated by Tip60 nor did they interact with Tip60. Fe65 is again unique in this aspect.

We performed mass spectrometry analysis to identify the Fe65 lysine residues acetylated by Tip60. This method detected several lysine residues, of which the most frequently acetylated sites were K204 and K701. Sitedirected mutagenesis of Fe65 confirmed that mutation of Fe65 K204 and K701 to arginine abolished the acetylation signal. In addition, mutation of all lysine residues to arginine, except K204 and K701, still allowed acetylation of Fe65. We analyzed the capacity of the acetylation-defective Fe65 mutant to form AFT spots together with APP and Tip60. Although $\triangle$ KAT Tip60 does not promote AFT spot formation, Fe65 mutation of K204 and K701 to arginine or glutamine did not prevent AFT spot formation with wt Tip60. Even simultaneous mutation of all lysine residues to arginine in Fe65 still enabled APP/Tip60 binding and AFT spot formation. This shows a great tolerance of Fe65 towards lysine to arginine switching, which maintains the positive charge, and proposes that steric changes induced by putative acetylation of lysine residues are not essential for AFT spot formation.

To directly study the effects of acetylation on protein structure, one would have to incorporate an acetylated lysine into Fe65, for instance using synthetically-evolved acetyl-lysyl-tRNA-synthetase/tRNA pairs (Umehara et al. 2012; Willis and Chin 2018). To study our cellular readouts of protein interaction, AFT spot formation and transcription, we genetically encoded the incorporation of arginine and glutamine residues in place of lysine, which serve as mimics of acetylation. Arginine exchange more closely resembles the steric changes with acetylation, whereas glutamine substitution mimics the charge neutralization but poorly the steric changes. Lysine 204 and 701 are located in unstructured regions of Fe65, therefore steric changes induced by acetylation might not have much effect. We have recently shown that a negatively charged acidic motif in Fe65, which precedes the WW domain, contributes to transcriptional activity (Probst et al. 2020). Lysine 204 is located in the linker between the acidic motif and the also transcriptionally active WW domain. It is possible that removal of the positive charge of K204 by acetylation improves their interaction with the transcription machinery. K701 at the Fe65 C-terminus is located in a region shown to be involved in intramolecular interaction between the WW domain and C-terminal parts of Fe65, resulting in a putatively inactive closed confirmation (Cao and Sudhof 2004). We have shown that deletion of the C-terminus increases Fe65 transcriptional activity (Probst et al. 2020), which would fit such a model. Acetylation and charge neutralization in the C-terminus could also disrupt interaction with the WW domain. Taken together, we conclude that the removal of a positive charge in K204 and K701 could enable an opening of inactive Fe65, leading to increased transcriptional activity. Due to the lack of a full-length Fe65 structure, we yet do not have a molecular model of how the AFT complex interacts with DNA and the exact effects of acetylation.

We did not detect acetylation of APP/AICD and Fe65 acetylation is not required for AFT spot formation. The autoacetylation of Tip60 or acetylation of other substrates might thus be required for AFT spot formation. Coexpression of differently tagged wt and $\triangle$ KAT Tip60 together with Fe65 resulted in the relocation of $\triangle \mathrm{KAT}$ Tip60 from speckles to spots. These data show that Tip60 autoacetylation can be conducted by an intermolecular acetylation step and that this acetylation is sufficient to enable relocation. In conclusion, Tip60 autoacetylation is necessary for the relocation from nuclear speckles to AFT spots, probably related to a different interaction with Fe65. This is in line with studies showing that Tip60 forms oligomers and autoacetylation causes their dissociation. This enhances Tip60 binding to its substrates, increasing their acetylation levels (Wang and Chen 2010; Xiao et al. 2014). Combined with our data this could imply that Tip60 localized to speckles is in an oligomeric form with autoacetylation being a prerequisite for oligomer dissociation, Fe65 binding and spot relocation. The importance of Tip60 autoacetylation for interaction with Fe65 is also supported by our half-life measurements. Both wt and $\triangle$ KAT Tip60 had a half-life of approximately $2 \mathrm{~h}$, which is in the reported range (Legube et al. 2002). The $\triangle \mathrm{KAT}$ mutant has been reported to display reduced protein levels and enhanced ubiquitination levels (Xiao et al. 2014). We detected only a small, non-significant change in half-life of wt and $\Delta K A T$ Tip60, but co-expression of Fe65 more than doubled the 
half-life of wt Tip60, having no effect on the $\Delta$ KAT mutant. Together with our finding of less co-precipitation of Fe65 with $\triangle$ KAT Tip60, we propose that the interaction with Fe65 stabilizes Tip60. Thus, the interaction of Fe65 with Tip60, as well as the downstream effects of Tip60 stabilization and spot relocation of both, depend on the prior autoacetylation of Tip60. In contrast to Tip60, Fe65 is a relatively stable protein with a half-life of around $25 \mathrm{~h}$ (Probst et al. 2020). We detected no effect of co-expressing either wt or $\triangle \mathrm{KAT}$ Tip60 on Fe65 half-life. Along those lines, the Fe65 K204/701R mutant that cannot be acetylated by Tip60 also had an unaltered half-life, proposing that these lysine residues are not a target of ubiquitination (data not shown).

How unique is Tip60 in mediating AICD-Fe65 signaling? Several KATs have common acetylation targets. An example is p53 acetylation at K120 by the MYST KATs Tip60 and its closest homolog, hMOF (Li et al. 2009; Neal et al. 2000; Rea et al. 2007; Sykes et al. 2006). We demonstrated activity of both MYST KATs by detecting the acetylation of p53. Nevertheless, we could not detect acetylation of Fe65 by hMOF. There are more than 20 different KAT enzymes described to date (Narita et al. 2019). It is also possible that non-enzymatic acetylation could regulate Fe65, but this occurs mainly in alkaline environments such as the mitochondrial matrix, where Fe65 is not localized. Analyzing members of the two other main KAT families, we saw no Fe65 acetylation by GCN5, but Fe65 was acetylated by CBP. We showed that CBP acetylates Fe65 at lysine residues different from K204/701. Furthermore, CBP co-expression increased Fe65 levels 3-5-fold. We conclude that the lysine residues targeted by CBP are probably targets for ubiquitination, as opposed to K204/701 targeted by Tip60.

We have reported that Fe65 has an extensive interaction interface with Tip60 (Probst et al. 2020). Of the tested KATs, only Tip60 was co-precipitated with Fe65, not hMOF or CBP. Furthermore, only Tip60 forms nuclear spots with Fe65 without further components transfected. Therefore, the KAT Tip60 has unique functions in AICD-Fe65 transcriptional signaling.

Acetylation levels are regulated by an interplay of KATs and KDACs. Sirt1 was shown to deacetylate Tip60, thereby reducing its KAT activity (Peng et al. 2012; Wang and Chen 2010). Expression of wt Sirt1 only nonsignificantly reduced Tip60 acetylation levels in our hands. We also did not detect a reduction of Fe65-Tip60 interaction, which would result from Tip60 deacetylation. Still, we could detect a significant decrease of Fe65 acetylation levels by Sirt1 expression, proposing that Fe65 is a direct substrate for Sirt1. In line with an involvement of Sirt1, its activation by resveratrol decreases Fe65 transcriptional activity. This can be counteracted by co-expression of Tip60. Finally, inhibition of class III KDACs with NAM increased transcriptional activity of Fe65.

$\mathrm{NAD}^{+}$levels decline in aging brain and neurodegenerative diseases (Lautrup et al. 2019; Zhu et al. 2015), causing reduced activity of the class III KDAC Sirt1. Loss of Sirt1 activity results in increased BACE expression, promoting amyloidogenic cleavage of APP (Wang et al. 2013). Sirt1 expression or activation with resveratrol was shown to be neuroprotective in mouse models of AD (Kim et al. 2007). Nuclear signaling by AICD/Fe65 is mainly mediated by the amyloidogenic cleavage pathway (Belyaev et al. 2010; Flammang et al. 2012; Goodger et al. 2009), thus nuclear signaling is increased by loss of Sirt1 activity. Additionally, we show that loss of Sirt1 activity can lead to increased Fe65 acetylation and thus increased transcriptional activity. These combined effects will potentiate Fe65-mediated gene expression that might play a role in neurodegeneration in AD (Konietzko 2012).

Transcriptional activity of Fe65 could also be enhanced by inhibition of class I/II KDACs with TSA, which was more evident when performing the UAS-Cit assay in the linear range. In parallel to enhanced activity we also detected enhanced Fe65 levels, which could explain why changes are difficult to detect in the saturated range of plasmid transfection.

Thus, both CBP expression and class I/II KDAC inhibition increase Fe65 levels, suggesting that both target the same lysine residues-differing from K204/701-to regulate ubiquitination. In addition, it is possible that class I/II KDAC inhibition has indirect effects via increased Tip60 acetylation.

Pharmacological inhibition with a specific Tip60 inhibitor (Gao et al. 2014) strongly reduced, while class III KDAC inhibition increased transcriptional activity. None of those treatments affected Fe65 protein levels, thus probably represent acetylation-dependent conformational changes that influence protein activity.

In a recent study we have reported evidence suggesting that Fe65 is a transcription factor (Probst et al. 2020). We now used our Gal4/UAS-Cit transcription assay (Konietzko et al. 2019), to reveal that Fe65 acetylation regulates its transcriptional activity. Mutation of K204 and K701 to arginine residues resembles steric changes induced by acetylation but introduces a permanent positive charge that cannot be erased by acetylation. This Fe65 construct revealed a significant reduction in transcriptional activity. Changing the two lysine residues to glutamine strongly increased transcription compared to wt Fe65. The switch to glutamine permanently removes the positive charge of lysine and mimics charge neutralization by acetylation. We conclude 
that acetylation of Fe65 by Tip60 functions to increase transcriptional activity, most likely due to the removal of a positive charge. Due to the state of current techniques, we were not able to study endogenous proteins and determine the stoichiometry of acetylation under in situ conditions. Nevertheless, because we could manipulate transcriptional activity of transfected Fe65 in both directions, we propose that regulation of Fe65 acetylation levels to control transcription occurs also in vivo. Acetylation does not act as an on/off switch for transcriptional activity but has a modulatory function, as described for other acetylation events. CBP-mediated acetylation enhances p53 basal transcriptional activity (Gu et al. 1997) and the complete deacetylation of Tip60 with SIRT1-detected by Western blotting with anti-acetyl-lysine antibodies-only leads to a 30\% reduction in KAT activity, not a complete block (Yang et al. 2012).

Acetylation of proteins in the nucleus is generally associated with transcriptional activation. Accordingly, we have shown that Tip60 acetylates Fe65 and an acetylationmimicking Fe65 mutant has increased transcriptional activity. Nevertheless, co-expression of wt Tip60 with Gal4Fe65 in our transcription assay reduced Fe65 transcriptional activity and the same was true for the Gal4-Fe65 K204/701R and K204/701Q mutants. Co-expression of the $\triangle$ KAT Tip60 mutant in contrast, had no effect on transcriptional activity of the Fe65 variants. The Fe65 K204/701Q mutant showed increased transcriptional activity compared to wt Fe65 that was similarly reduced by wt Tip60 co-expression. Two other publications have also shown repressive effects of Tip60 in luciferase assays with Fe65-Gal4 and APP-Gal4, and came up with different explanations for this unexpected finding. Either this effect was attributed to inefficient recruitment of overexpressed Tip60 to the reporter gene, causing trapping of other components of the Tip60 multimolecular complex away from the reporter gene (Scheinfeld et al. 2003), or it was concluded that Tip60 is an Fe65-associated repressor (Yang et al. 2006). We present a more detailed insight into the mechanism of Tip60-mediated reduction in Fe65 transcriptional activity that favors the first explanation. We have consistently observed the formation of spherical spots in the nucleus when Fe65 is co-expressed with Tip60 that also include AICD (von Rotz et al. 2004). These AFT spots mark the sites of transcription of endogenous target genes (Konietzko et al. 2010). The UAS-Cit transcription assay works with genome-integrated reporter genes (Konietzko et al. 2019). In a recent paper (Probst et al. 2020), we showed that the reporter gene integration sites do not localize to AFT spots but to nuclear speckles. Consequently, when Tip60 coexpression results in relocation of Gal4-Fe65 to spots, it is depleted from the sites of reporter gene integration and thus transcriptional activity readout is reduced. This is in line with the fact that the $\triangle$ KAT Tip60 mutant does not support the formation of AFT spots and also does not lower the Fe65 transcriptional activity readout. Similar effects are expected to occur when luciferase reporter plasmids are transiently transfected (Scheinfeld et al. 2003; Yang et al. 2006), coexpression of Tip60 will relocate Gal4-Fe65 away from the reporter plasmids and thus cause a reduced transcription readout. Therefore, our conclusion is that Tip60 has a positive effect on Fe65-mediated transcription, by localizing Fe65 to nuclear spots and increasing its transcriptional activity. The putatively negative effect of Tip60 on Fe65mediated transcription is related to the technical details of the transcription assays and is misleading.

We report for the first time the regulation of Fe65 transcriptional activity by acetylation via two different acetylation/deacetylation cycles (Figure 7E). Tip60 acetylates K204 and K701 to increase activity, which is counteracted by class III KDACs, specifically Sirt1. K204/701 acetylation does not affect Fe65 turnover, thus probably causes conformational changes enabling improved transcriptional activity. Another acetylation cycle, conducted by CBP and class I/II KDACs, targets lysine residues different from K204/701 that are also probable targets of ubiquitination, as their acetylation stabilizes Fe65 protein levels. Of the analyzed KATs, only Tip60 strongly binds Fe65 and leads to relocation of Fe65 to nuclear spots. The autoacetylation of Tip60 is crucial for the dissolvement of Tip60 speckles and the formation of AFT spots at sites of transcription of endogenous target genes. Thus, acetylation can modulate the nuclear signaling and transcription initiated by APP processing by regulating both Tip60 and Fe65 functions.

\section{Materials and methods}

\section{DNA constructs}

The following plasmids have been described previously: Cit-AICD, APP695-Cit, HA-Fe65, CFP-Tip60 (human, isoform 2), CFP-NLS (von Rotz et al. 2004); myc-Tip60 (Konietzko et al. 2010); APP695-Cer (Gersbacher et al. 2013); APP695-YC155 and myc-Tip60-YN155 (Riese et al. 2013); streptavidin-binding peptide (SBP)-myc-Fe65 (Probst et al. 2020). Wt and H363Y FLAG-Sirt1 were obtained from Addgene (\#1791 and \#1792, deposited by Michael Greenberg) (Brunet et al. 2004). A myc-tagged hMOF was obtained from Genscript (OHu23171C, Piscataway, USA). It was cloned into the pcDNA3.1+/ $\mathrm{C}$-Myc vector using $\mathrm{KpnI}$ and $\mathrm{XbaI}$ restriction sites by to generate hMOF-myc. GFP-p53 (\#12091, Addgene, deposited by Tyler Jacks) (Boyd et al. 2000) was used to PCR amplify p53, which was fused to a $\mathrm{N}$-terminal SBP-myc tag and cloned into the pUKBK vector system (Kohli et al. 2012) to create SBP-myc-p53. From Addgene, we obtained GFP-GCN5 (\#65386, deposited by Kyle Miller) and FLAG-CBP-HA 
(\#32908, deposited by Tso-Pang Yao). Myc-Tip60 Q377E and mycTip60 G380E were generated by site-directed mutagenesis. To generate $\triangle$ KAT Tip60 constructs, both Q377E and G380E mutations were introduced into myc-Tip60, myc-Tip60-YN155, and CFP-Tip60. mCherry was obtained by PCR from a mCherry-CI-RelA construct and fused to Fe65 to generate mCherry-Fe65 (mChe-Fe65) and Cerulean was fused to generate Cer-Fe65. SBP-myc-Fe65L1 (mouse), SBP-mycFe65L2 (human), and Gal4-myc-Fe65 have been described (Probst et al. 2020). Lysine to arginine and lysine to glutamine mutations were introduced into the SBP-myc-Fe65 and Gal4-myc-Fe65 constructs by site-directed mutagenesis, generating K204R, K701R, K204/701R, K204Q, K701Q, and K204/701Q mutants. Multi-site-directed mutagenesis using the QuikChange Multi Site-Directed Mutagenesis Kit (\#200515, Agilent) was applied to mutate all 25 lysine residues as well as the two lysine residues in the SBP and in the myc tag to arginine, generating SBP-myc-Fe65 'all KtoR' (K-50/-11/39/48/68/71/111/152/ 204/318/332/357/368/405/420/432/472/478/489/501/522/536/542/ 555/660/701/703 with K-50 and K-11 representing lysine residues in the SBP- and myc-tag, respectively). SBP-myc-Fe65 'all KtoR, except K204 and K701' was generated by mutating R204 and R701 of the SBP-myc-Fe65 'all KtoR' plasmid back to lysine residues. Details of oligonucleotide primers and mutagenesis strategy are shown in Supplementary Tables S1 and S2. Fe65 K to R and K to Q mutants were excised by restriction enzymes from SBP-myc vectors and fused to mCherry or the Gal4-DBD using the pUKBK vector system (Kohli et al., 2012).

\section{Cell culture}

N2a (DSM, Braunschweig, Germany) and human embryonic kidney cells (HEK293; DSMZ, Braunschweig, Germany) cells were maintained in Dulbecco's modified eagle's medium (DMEM; \#41965039, ThermoFisher Scientific) supplemented with $10 \%$ fetal bovine serum (FBS; \#10270106, ThermoFisher Scientific) under standard conditions ( $37^{\circ} \mathrm{C}, 5 \% \mathrm{CO}_{2}$, humified atmosphere). For confocal microscopy, cells were cultured on Falcon ${ }^{\circledR}$ 4-well culture slides (\#354104, Corning $\mathrm{GmbH}$, Kaiserslautern, Germany), sequentially coated with $50 \mu \mathrm{g} / \mathrm{ml}$ poly-L-ornithine (\#P3655, Sigma) and $5 \mu \mathrm{g} / \mathrm{ml}$ fibronectin (\#F4759, Sigma). For lipofection with Lipofectamine ${ }^{\mathrm{TM}} 2000$ Transfection Reagent (\#11668019, ThermoFisher Scientific) the manufacturer's protocol was followed. After 20-24 h, cells were fixed with 4\% paraformaldehyde or lysed with homogenization buffer for pulldown experiments (see below) or, in the case of CHX experiments, RIPA buffer (50 mM Tris- $\mathrm{HCl} \mathrm{pH}$ 7.6, $150 \mathrm{mM} \mathrm{NaCl}, 1 \%$ NP40, 0.1\% SDS, 0.5\% sodium deoxycholate, 2 mM EDTA, 1 tablet EDTA-free protease inhibitor cocktail / 50 ml (\#04693159001, Sigma Aldrich). For pharmacological treatments, the medium was replaced with fresh culture medium containing $100 \mathrm{nM}$ resveratrol (\#R5010, Sigma), $50 \mu \mathrm{M}$ or $100 \mu \mathrm{M}$ Tip60 KAT inhibitor TH1834 (\#2339, Axon), $500 \mathrm{nM}$ or $1 \mu \mathrm{M}$ KDAC inhibitor Trichostatin A (\#S1045, Selleckchem), 5 mM Nicotinamide (\#N0636, Sigma), or 0.3\% DMSO as solvent control, $2.5 \mathrm{~h}$ following transfection. Cells were then cultured for another $21.5 \mathrm{~h}$ before fixation.

\section{Immunocytochemistry and antibodies}

Transfected HEK293 and N2a cells were fixed with 4\% PFA in phosphate-buffered saline (PBS) and blocked with Tris-buffered saline (TBS) containing $0.2 \%$ Triton, $5 \%$ goat serum and 5\% horse serum.
All antibody incubations and wash steps were performed with TBS. Primary antibodies were c-myc (mouse IgG1K, 9E10, monoclonal, 1:100, \#11 667149 001, Roche), mCherry (rat IgG2a, 16D7, monoclonal, 1:2000, \#M11217, ThermoFisher) and HA high affinity (rat IgG1, 3F10, monoclonal, 1:100, \#11 867431 001, Roche). The following highly cross-purified secondary antibodies were purchased from Jackson ImmunoResearch and used at a concentration of 1:250: DyLight ${ }^{\mathrm{TM}}$

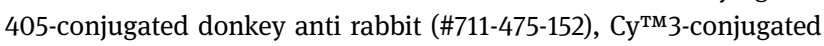
donkey anti mouse (\#715-165-151), Су СМ3-conjugated donkey anti rat (\#712-165-153), Alexa Fluor $^{\circledR}$ 647-conjugated donkey anti mouse (\#715-605-151), Alexa Fluor ${ }^{\circledR}$ 647-conjugated donkey anti rat (\#712-605-153), and Cy ${ }^{\mathrm{TM} 5}$-conjugated donkey anti rat (\#712-175-153). Nuclei were counterstained with DAPI, and cells were mounted with Mowiol containing 2.5\% DABCO (1,4-diazabicyclo[2.2.2]octane).

\section{Confocal microscopy}

Images were acquired on a TCS/SP8 confocal microscope (Leica, Wetzlar, Germany). For the BiFC experiment and for the analysis of AFT spot formation, all dyes were sequentially excited and detected, except for DAPI and Cy5/AlexaFluor 647 which were imaged simultaneously. The $405 \mathrm{~nm}$ laser was used to excite DyLight 405 (HyD window: 405-450 nm) and DAPI (HyD window: 405-450 nm), the $442 \mathrm{~nm}$ laser to excite CFP (HyD window: 445-500 nm), the $514 \mathrm{~nm}$ laser line of the argon laser for the BiFC signal (yellow fluorescent protein, YFP) (HyD window: 515-550 nm) and Cit (HyD window: 515-590 nm), the $561 \mathrm{~nm}$ laser for Cy3 (HyD window: 560-610 nm), the $633 \mathrm{~nm}$ laser for Cy5/Alexa Fluor 647 (HyD window: 635-680 nm for $\mathrm{BiFC}$ signal, or $650-710 \mathrm{~nm}$ for AFT spots). All images are maximum projections of five to ten $\mathrm{z}$-sections acquired with a $63 \mathrm{x}$ glycerol immersion objective. For the analysis of transactivation assays, single sections were acquired with a 20x glycerol immersion objective as described (Konietzko et al. 2019). Secondary antibody-coupled dyes and fluorescent proteins were coded in the same colors in all pictures: DyLight 405 (magenta), Cerulean/CFP (cyan), Cit (yellow), Cy3 and mCherry (red), Alexa 647 (blue), and DAPI (white).

\section{Transactivation assay}

The transactivation assay was performed as described (Konietzko et al. 2019). Briefly, Fe65 protein constructs with an N-terminal Gal4-DBD-myc tag (Probst et al. 2020) were transfected into HEK2:UAS-Cit-NLS cells. Except for Figure 6E, F, where 150 ng DNA was transfected, $1 \mu \mathrm{g}$ was used in all other transcription assays. Approximately $22 \mathrm{~h}$ post-transfection, cells were fixed and counterstained with DAPI and anti-myc, followed by anti-mouse Alexa Fluor ${ }^{\circledR} 647$. Seven confocal images were taken of each well and experiments repeated with different cell passages. The analysis was performed using a custom-written ImageJ Macro to detect Cit levels in the nuclei and total Alexa Fluor ${ }^{\circledR} 647$ fluorescence, with normalization of the signals by the number of detected DAPI-stained cells.

\section{Dynabeads-Streptavidin-based pulldown assays}

Pulldown of SBP-tagged constructs was performed with DynabeadsStreptavidin. Approximately $22 \mathrm{~h}$ following transfection, HEK293 cells were lysed by passing through a $26 \mathrm{G}$ needle in homogenization buffer (140 mM KCl, 20 mM HEPES pH 7.2, 10 mM NaCl, 5\% [v/v] glycerol, 
$2 \mathrm{mM} \mathrm{MgSO}_{4}, 1 \%$ [v/v] Triton-X100, 2 mM DTT, EDTA-free ProteaseInhibitor Cocktail [\#11873580001, Roche], and $2 \mathrm{mM}$ phenantrolene). After incubation on a rotating wheel for $15 \mathrm{~min}$ at $4{ }^{\circ} \mathrm{C}$ and clearance by centrifugation at $800 \mathrm{~g}$ for $10 \mathrm{~min}$ at $4^{\circ} \mathrm{C}$, the supernatant was incubated with Dynabeads ${ }^{\circledR}$ M-280 Streptavidin (\#11205D, ThermoFisher Scientific) for $4 \mathrm{~h}$ at $4^{\circ} \mathrm{C}$. The beads were washed and the bound proteins were eluted with $14 \mathrm{mM}$ biotin.

\section{Western blot analysis}

Equal volumes of protein samples from the pulldown experiments were separated on Novex ${ }^{\mathrm{TM}} 10-20 \%$ Tricine Protein Gels (\#EC6625BOX, ThermoFisher Scientific) and transferred on a $0.1 \mu \mathrm{m}$ Amersham $^{\mathrm{TM}}$ Protran $^{\mathrm{TM}}$ nitrocellulose blotting membrane (\#GE10600000, GE Healthcare). The blots were incubated with following primary antibodies: acetylated lysine (acK; rabbit, polyclonal, 1:1000, \#9441, Cell Signaling), APP C-term (rabbit IgG, Y188, monoclonal, 1:250, \#ab32136, Abcam), c-myc (mouse $\operatorname{IgG}_{1} \mathrm{~K}$, 9E10, monoclonal, 1:1000, \#11 667149 00, Roche), FLAG (rabbit IgG, polyclonal, 1:1000, \#F7425, Sigma), GAPDH (mouse $\operatorname{IgG}_{1}$, monoclonal, 1:5000, \#H86504M, Meridian), GFP (mouse $\mathrm{IgG}_{1} \mathrm{~K}$, clones 7.1 and 13.1, 1:1000, \#11 814460 001, Roche), HA high affinity (rat IgG1, 3F10, monoclonal, 1:1000, \#11 867431 001, Roche), 6x-His (mouse lgG2a, monoclonal, 1:4000, \#631212, Takara), p53 acetyl K120 (mouse IgG1 kappa, 10E5, monoclonal, 1:1000, \#ab78316, Abcam), and Sirt1 (rabbit IgG, E54, monoclonal, 1:1000, \#ab32424, Abcam). Secondary antibodies were peroxidase-linked goat anti mouse (1:2500; \#115-035-146, Jackson ImmunoResearch), peroxidase-linked goat anti rabbit (1:2500, \#115-035-045, Jackson ImmunoResearch), and peroxidaselinked goat anti rat (1:2500, \#NA935, GE Healthcare). ECL detection was performed with the ImageQuant LAS 4000 (GE Healthcare Life Sciences) using Pierce ${ }^{\mathrm{TM}}$ ECL Western Blotting Substrate (\#32106, ThermoFisher Scientific) or ECL Prime Western Blotting Detection Reagent (\#RPN2232, GE Healthcare). The ImageQuant TL software was used to quantify the latest exposure before the brightest band was saturated on the blot. For the half-life experiments the protein levels were normalized to GAPDH, and fitted to the exponential model $f(x)=a^{\star} e^{(b x)}$ using Matlab R2014b. The half-life equals to (2)/-b.

\section{Statistical analysis}

Statistics were calculated with SPSS version 23. Data were analyzed for normal distribution by Shapiro-Wilk test, followed by two-tailed Student's $t$-test or Mann-Whitney $U$ test against control or wild type. Figures 6 and 7 were analyzed with Prism9. Normal distribution was determined by Shapiro-Wilk test. Normal distributed data were analyzed by one-way ANOVA with Bonferroni or Tukey's correction. Not normal distributed data were analyzed by Kruskal-Wallis with Dunn's correction. Asterisks above the horizontal significance line refer to all bars with ticks except when the ticks are marked individually. $p<0.1(\#), p<0.05\left(^{\star}\right), p<0.01\left(^{\star \star}\right)$ and $p<0.001\left(^{\star \star \star}\right)$. Bars not indicated by a tick are not significantly different.

\section{Mass spectrometry}

HEK293 cells were co-transfected with SBP-myc-Fe65 and wt or $\triangle$ KAT myc-Tip60. The lysates were subjected to Dynabeads-Streptavidinbased pulldowns, and eluated proteins were separated on a Novex ${ }^{\mathrm{TM}}$
10-20\% Tricine Protein Gel (\#EC6625BOX, ThermoFisher Scientific). After Coomassie staining, gel bands around $80 \mathrm{kDa}$ were excised, digested with trypsin, and extracted with $0.1 \%$ TFA (trifluoroacetic acid) $/ 50 \%$ acetonitrile. Supernatants were dried, dissolved in $0.1 \%$ formic acid (Romil) and analyzed on a nanoAcquity UPLC (Waters Inc.) connected to a Q Exactive mass spectrometer (ThermoFisher Scientific). De novo sequencing and search against a database containing only the expected sequences was performed using Peaks. For MALDI-MS/MS (matrix assisted laser desorption/ionization-MS/MS), the sample was mixed 1:1 with matrix solution $(0.7 \mathrm{mg} / \mathrm{ml} \mathrm{HCCA}$ in $85 \% \mathrm{ACN}, 0.1 \% \mathrm{TFA}$, $1 \mathrm{mMNH}_{4} \mathrm{H}_{2} \mathrm{PO}_{4}$ ), spotted on the target, desalted, and concentrated with $0.1 \%$ TFA. Measurements were performed with the Ultraflextreme MALDI TOF-TOF instrument (Bruker) in reflect mode. The data were analyzed with BioTools Version 3.2 (Bruker) and a multiple spectra report was created with the Bruker Daltonics flexAnalysis (Bruker).

Acknowledgments: We thank Peter Hunziker from the Functional Genomics Center Zürich for his support with the mass spectrometry.

Author contributions: All the authors have accepted responsibility for the entire content of this submitted manuscript and approved submission.

Research funding: This work was supported by the Swiss National Science Foundation SNF 31003A_166177 and SNF 31003A_146532 as well as the resources of the IREM.

Conflict of interest statement: The authors declare no competing or financial interests.

\section{References}

Baek, S.H., Ohgi, K.A., Rose, D.W., Koo, E.H., Glass, C.K., and Rosenfeld, M.G. (2002). Exchange of N-CoR corepressor and Tip60 coactivator complexes links gene expression by NF-KB and $\beta$-amyloid precursor protein. Cell 110: 55-67.

Belyaev, N.D., Kellett, K.A., Beckett, C., Makova, N.Z., Revett, T.J., Nalivaeva, N.N., Hooper, N.M., and Turner, A.J. (2010). The transcriptionally active amyloid precursor protein (APP) intracellular domain is preferentially produced from the 695 isoform of APP in a $\beta$-secretase-dependent pathway. J. Biol. Chem. 285: 41443-41454.

Boyd, S.D., Tsai, K.Y., and Jacks, T. (2000). An intact HDM2 RING-finger domain is required for nuclear exclusion of p53. Nat. Cell Biol. 2: 563-568.

Brady, M.E., Ozanne, D.M., Gaughan, L., Waite, I., Cook, S., Neal, D.E., and Robson, C.N. (1999). Tip60 is a nuclear hormone receptor coactivator. J. Biol. Chem. 274: 17599-17604.

Brunet, A., Sweeney, L.B., Sturgill, J.F., Chua, K.F., Greer, P.L., Lin, Y., Tran, H., Ross, S.E., Mostoslavsky, R., Cohen, H.Y., et al. (2004). Stress-dependent regulation of FOXO transcription factors by the SIRT1 deacetylase. Science 303: 2011-2015.

Cao, X. and Sudhof, T.C. (2001). A transcriptionally [correction of transcriptively] active complex of APP with Fe65 and histone acetyltransferase Tip60. Science 293: 115-120.

Cao, X. and Sudhof, T.C. (2004). Dissection of amyloid- $\beta$ precursor protein-dependent transcriptional transactivation. J. Biol. Chem. 279: 24601-24611. 
Cho, W.K., Spille, J.H., Hecht, M., Lee, C., Li, C., Grube, V., and Cisse, II. (2018). Mediator and RNA polymerase II clusters associate in transcription-dependent condensates. Science 361: 412-415.

Choudhary, C., Kumar, C., Gnad, F., Nielsen, M.L., Rehman, M., Walther, T.C., Olsen, J.V., and Mann, M. (2009). Lysine acetylation targets protein complexes and co-regulates major cellular functions. Science 325: 834-840.

Cui, H., Guo, M., Xu, D., Ding, Z.C., Zhou, G., Ding, H.F., Zhang, J., Tang, Y., and Yan, C. (2015). The stress-responsive gene ATF3 regulates the histone acetyltransferase Tip60. Nat. Commun. 6: 6752.

Donmez, G., Wang, D., Cohen, D.E., and Guarente, L. (2010). SIRT1 suppresses beta-amyloid production by activating the $\alpha$-secretase gene ADAM10. Cell 142: 320-332.

Duilio, A., Zambrano, N., Mogavero, A.R., Ammendola, R., Cimino, F., and Russo, T. (1991). A rat brain mRNA encoding a transcriptional activator homologous to the DNA binding domain of retroviral integrases. Nucleic Acids Res. 19: 5269-5274.

Feilen, L.P., Haubrich, K., Strecker, P., Probst, S., Eggert, S., Stier, G., Sinning, I., Konietzko, U., Kins, S., Simon, B., et al. (2017). Fe65-PTB2 dimerization mimics Fe65-APP interaction. Front. Mol. Neurosci. 10: 140.

Flammang, B., Pardossi-Piquard, R., Sevalle, J., Debayle, D., DabertGay, A.S., Thevenet, A., Lauritzen, I., and Checler, F. (2012). Evidence that the amyloid- $\beta$ protein precursor intracellular domain, AICD, derives from $\beta$-secretase-generated $C$-terminal fragment. J. Alzheim. Dis. 30: 145-153.

Gao, C., Bourke, E., Scobie, M., Famme, M.A., Koolmeister, T., Helleday, T., Eriksson, L.A., Lowndes, N.F., and Brown, J.A. (2014). Rational design and validation of a Tip60 histone acetyltransferase inhibitor. Sci. Rep. 4: 5372.

Gersbacher, M.T., Goodger, Z.V., Trutzel, A., Bundschuh, D., Nitsch, R.M., and Konietzko, U. (2013). Turnover of amyloid precursor protein family members determines their nuclear signaling capability. PloS One 8: e69363.

Goodger, Z.V., Rajendran, L., Trutzel, A., Kohli, B.M., Nitsch, R.M., and Konietzko, U. (2009). Nuclear signaling by the APP intracellular domain occurs predominantly through the amyloidogenic processing pathway. J. Cell Sci. 122: 3703-3714.

Grunstein, M. (1997). Histone acetylation in chromatin structure and transcription. Nature 389: 349-352.

Gu, W., Shi, X.L., and Roeder, R.G. (1997). Synergistic activation of transcription by CBP and p53. Nature 387: 819-823.

Ikura, T., Ogryzko, V.V., Grigoriev, M., Groisman, R., Wang, J., Horikoshi, M., Scully, R., Qin, J., and Nakatani, Y. (2000). Involvement of the TIP60 histone acetylase complex in DNA repair and apoptosis. Cell 102: 463-473.

Kamine, J., Elangovan, B., Subramanian, T., Coleman, D., and Chinnadurai, G. (1996). Identification of a cellular protein that specifically interacts with the essential cysteine region of the HIV-1 Tat transactivator. Virology 216: 357-366.

Kang, J., Lemaire, H.G., Unterbeck, A., Salbaum, J.M., Masters, C.L., Grzeschik, K.H., Multhaup, G., Beyreuther, K., and Muller-Hill, B. (1987). The precursor of Alzheimer's disease amyloid A4 protein resembles a cell-surface receptor. Nature 325: 733-736.

Kelley, L.A., Mezulis, S., Yates, C.M., Wass, M.N., and Sternberg, M.J. (2015). The Phyre2 web portal for protein modeling, prediction and analysis. Nat. Protoc. 10: 845-858.

Kim, D., Nguyen, M.D., Dobbin, M.M., Fischer, A., Sananbenesi, F., Rodgers, J.T., Delalle, I., Baur, J.A., Sui, G., Armour, S.M., et al. (2007). SIRT1 deacetylase protects against neurodegeneration in models for Alzheimer's disease and amyotrophic lateral sclerosis. EMBO J. 26: 3169-3179.

Kimura, A. and Horikoshi, M. (1998). Tip60 acetylates six lysines of a specific class in core histones in vitro. Gene Cell. 3: 789-800.

Kohli, B.M., Pflieger, D., Mueller, L.N., Carbonetti, G., Aebersold, R., Nitsch, R.M., and Konietzko, U. (2012). Interactome of the amyloid precursor protein APP in brain reveals a protein network involved in synaptic vesicle turnover and a close association with synaptotagmin-1. J. Proteome Res. 11: 4075-4090.

Konietzko, U. (2012). AICD nuclear signaling and its possible contribution to Alzheimer's disease. Curr. Alzheimer Res. 9: 200-216.

Konietzko, U., Gersbacher, M.T., Streuli, J., Kruger, M., Thoni, S., Kins, S., and Nitsch, R.M. (2019). A fluorescent protein-readout for transcriptional activity reveals regulation of APP nuclear signaling by phosphorylation sites. Biol. Chem. 400: 1191-1203.

Konietzko, U., Goodger, Z.V., Meyer, M., Kohli, B.M., Bosset, J., Lahiri, D.K., and Nitsch, R.M. (2010). Co-localization of the amyloid precursor protein and Notch intracellular domains in nuclear transcription factories. Neurobiol. Aging 31: 58-73.

Lautrup, S., Sinclair, D.A., Mattson, M.P., and Fang, E.F. (2019). NAD ${ }^{+}$ in brain aging and neurodegenerative disorders. Cell Metabol. 30: 630-655.

Lee, D.Y., Hayes, J.J., Pruss, D., and Wolffe, A.P. (1993). A positive role for histone acetylation in transcription factor access to nucleosomal DNA. Cell 72: 73-84.

Lee, K.K. and Workman, J.L. (2007). Histone acetyltransferase complexes: one size doesn't fit all. Nat. Rev. Mol. Cell Biol. 8: 284-295.

Legube, G., Linares, L.K., Lemercier, C., Scheffner, M., Khochbin, S., and Trouche, D. (2002). Tip60 is targeted to proteasomemediated degradation by Mdm2 and accumulates after UV irradiation. EMBO J. 21: 1704-1712.

Li, X., Wu, L., Corsa, C.A., Kunkel, S., and Dou, Y. (2009). Two mammalian MOF complexes regulate transcription activation by distinct mechanisms. Mol Cell 36: 290-301.

Narita, T., Weinert, B.T., and Choudhary, C. (2019). Functions and mechanisms of non-histone protein acetylation. Nat. Rev. Mol. Cell Biol. 20: 156-174.

Neal, K.C., Pannuti, A., Smith, E.R., and Lucchesi, J.C. (2000). A new human member of the MYST family of histone acetyl transferases with high sequence similarity to Drosophila MOF. Biochim. Biophys. Acta 1490: 170-174.

Pardossi-Piquard, R. and Checler, F. (2012). The physiology of the beta-amyloid precursor protein intracellular domain AICD. J. Neurochem. 120(Suppl 1): 109-124.

Peng, L., Ling, H., Yuan, Z., Fang, B., Bloom, G., Fukasawa, K., Koomen, J., Chen, J., Lane, W.S., and Seto, E. (2012). SIRT1 negatively regulates the activities, functions, and protein levels of hMOF and TIP60. Mol. Cell Biol. 32: 2823-2836.

Perez-Perri, J.I., Dengler, V.L., Audetat, K.A., Pandey, A., Bonner, E.A., Urh, M., Mendez, J., Daniels, D.L., Wappner, P., Galbraith, M.D., et al. (2016). The TIP60 complex is a conserved coactivator of HIF1A. Cell Rep. 16: 37-47.

Probst, S., Kruger, M., Kagi, L., Thoni, S., Schuppli, D., Nitsch, R.M., and Konietzko, U. (2020). Fe65 is the sole member of its family that mediates transcription regulated by the amyloid precursor protein. J. Cell Sci. 133, https://doi. org/10.1242/jcs.242917. 
Rea, S., Xouri, G., and Akhtar, A. (2007). Males absent on the first (MOF): from flies to humans. Oncogene 26: 5385-5394.

Riese, F., Grinschgl, S., Gersbacher, M.T., Russi, N., Hock, C., Nitsch, R.M., and Konietzko, U. (2013). Visualization and quantification of APP intracellular domain-mediated nuclear signaling by bimolecular fluorescence complementation. PloS One 8: e76094.

Roth, S.Y., Denu, J.M., and Allis, C.D. (2001). Histone acetyltransferases. Annu. Rev. Biochem. 70: 81-120.

Sapountzi, V., Logan, I.R., and Robson, C.N. (2006). Cellular functions of TIP60. Int. J. Biochem. Cell Biol. 38: 1496-1509.

Scheinfeld, M.H., Matsuda, S., and D'Adamio, L. (2003). JNK-interacting protein-1 promotes transcription of $A \beta$ protein precursor but not $A \beta$ precursor-like proteins, mechanistically different than Fe65. Proc. Natl. Acad. Sci. U.S.A. 100: 1729-1734.

Selkoe, D.J. and Hardy, J. (2016). The amyloid hypothesis of Alzheimer's disease at 25 years. EMBO Mol. Med. 8: 595-608.

Sun, Y., Jiang, X., Chen, S., Fernandes, N., and Price, B.D. (2005). A role for the Tip60 histone acetyltransferase in the acetylation and activation of ATM. Proc. Natl. Acad. Sci. U. S. A. 102: $13182-13187$.

Sykes, S.M., Mellert, H.S., Holbert, M.A., Li, K., Marmorstein, R., Lane, W.S., and McMahon, S.B. (2006). Acetylation of the p53 DNA-binding domain regulates apoptosis induction. Mol Cell 24: 841-851.

Tang, Y., Luo, J., Zhang, W., and Gu, W. (2006). Tip60-dependent acetylation of $\mathrm{p} 53$ modulates the decision between cell-cycle arrest and apoptosis. Mol Cell 24: 827-839.

Umehara, T., Kim, J., Lee, S., Guo, L.T., Soll, D., and Park, H.S. (2012). $\mathrm{N}$-acetyl lysyl-tRNA synthetases evolved by a CcdB-based selection possess $\mathrm{N}$-acetyl lysine specificity in vitro and in vivo. FEBS Lett. 586: 729-733.

von Rotz, R.C., Kohli, B.M., Bosset, J., Meier, M., Suzuki, T., Nitsch, R.M., and Konietzko, U. (2004). The APP intracellular domain forms nuclear multiprotein complexes and regulates the transcription of its own precursor. J. Cell Sci. 117: 4435-4448.

Wang, J.D. and Chen, J.J. (2010). SIRT1 regulates autoacetylation and histone acetyltransferase activity of TIP60. J. Biol. Chem. 285: 11458-11464.
Wang, R., Li, J.J., Diao, S., Kwak, Y.D., Liu, L., Zhi, L., Bueler, H., Bhat, N.R., Williams, R.W., Park, E.A., et al. (2013). Metabolic stress modulates Alzheimer's $\beta$-secretase gene transcription via SIRT1-PPARY-PGC-1 in neurons. Cell Metabol. 17: 685-694.

Wiley, J.C., Smith, E.A., Hudson, M.P., Ladiges, W.C., and Bothwell, M. (2007). Fe65 stimulates proteolytic liberation of the $\beta$-amyloid precursor protein intracellular domain. J. Biol. Chem. 282: 33313-33325.

Willis, J.C.W. and Chin, J.W. (2018). Mutually orthogonal pyrrolysyltRNA synthetase/tRNA pairs. Nat. Chem. 10: 831-837.

Xiao, Y., Nagai, Y., Deng, G.P., Ohtani, T., Zhu, Z.Q., Zhou, Z.C., Zhang, H.T., Ji, M.Q., Lough, J.W., Samanta, A., et al. (2014). Dynamic interactions between TIP60 and p300 regulate FOXP3 function through a structural switch defined by a single lysine on TIP60. Cell Rep. 7: 1471-1480.

Yang, C., Wu, J., and Zheng, Y.G. (2012). Function of the active site lysine autoacetylation in Tip60 catalysis. PloS One 7: e32886.

Yang, Z., Cool, B.H., Martin, G.M., and Hu, Q. (2006). A dominant role for FE65 (APBB1) in nuclear signaling. J. Biol. Chem. 281: 4207-4214.

Yi, J., Huang, X., Yang, Y., Zhu, W.G., Gu, W., and Luo, J. (2014). Regulation of histone acetyltransferase TIP60 function by histone deacetylase 3. J. Biol. Chem. 289: 33878-33886.

Zambrano, N., Buxbaum, J.D., Minopoli, G., Fiore, F., De Candia, P., De Renzis, S., Faraonio, R., Sabo, S., Cheetham, J., Sudol, M., et al. (1997). Interaction of the phosphotyrosine interaction/ phosphotyrosine binding-related domains of Fe 65 with wild-type and mutant Alzheimer's $\beta$-amyloid precursor proteins. J. Biol. Chem. 272: 6399-6405.

Zhao, S., Xu, W., Jiang, W., Yu, W., Lin, Y., Zhang, T., Yao, J., Zhou, L., Zeng, Y., Li, H., et al. (2010). Regulation of cellular metabolism by protein lysine acetylation. Science 327: 1000-1004.

Zhu, X.H., Lu, M., Lee, B.Y., Ugurbil, K., and Chen, W. (2015). In vivo NAD assay reveals the intracellular NAD contents and redox state in healthy human brain and their age dependences. Proc. Natl. Acad. Sci. U.S.A. 112: 2876-2881.

Supplementary Material: The online version of this article offers supplementary material (https://doi.org/10.1515/hsz-2020-0279). 